\title{
Bayesian reconstruction of gravitational wave burst signals from simulations of rotating stellar core collapse and bounce
}

\author{
Christian Röver, ${ }^{1}$ Marie-Anne Bizouard, ${ }^{2}$ Nelson Christensen, ${ }^{3}$ \\ Harald Dimmelmeier, ${ }^{4}$ Ik Siong Heng, ${ }^{5}$ and Renate Meyer ${ }^{6}$ \\ ${ }^{1}$ Max-Planck-Institut für Gravitationsphysik (Albert-Einstein-Institut), 30167 Hannover, Germany \\ ${ }^{2}$ Laboratoire de l'Accélérateur Linéaire, Université Paris Sud, 91898 Orsay, France \\ ${ }^{3}$ Physics and Astronomy, Carleton College, Northfield, Minnesota 55067, USA \\ ${ }^{4}$ Department of Physics, Aristotle University of Thessaloniki, 54124 Thessaloniki, Greece \\ ${ }^{5}$ Department of Physics and Astronomy, University of Glasgow, Glasgow G12 8QQ, UK \\ ${ }^{6}$ Department of Statistics, The University of Auckland, Auckland 1142, New Zealand
}

(Dated: July 3, 2021)

\begin{abstract}
Presented in this paper is a technique that we propose for extracting the physical parameters of a rotating stellar core collapse from the observation of the associated gravitational wave signal from the collapse and core bounce. Data from interferometric gravitational wave detectors can be used to provide information on the mass of the progenitor model, precollapse rotation and the nuclear equation of state. We use waveform libraries provided by the latest numerical simulations of rotating stellar core collapse models in general relativity, and from them create an orthogonal set of eigenvectors using principal component analysis. Bayesian inference techniques are then used to reconstruct the associated gravitational wave signal that is assumed to be detected by an interferometric detector. Posterior probability distribution functions are derived for the amplitudes of the principal component analysis eigenvectors, and the pulse arrival time. We show how the reconstructed signal and the principal component analysis eigenvector amplitude estimates may provide information on the physical parameters associated with the core collapse event.
\end{abstract}

PACS numbers: 02.70.Uu, 04.80.Nn, 05.45.Tp, 97.60.Bw

\section{INTRODUCTION}

The detection of gravitational radiation will likely come in the near future. LIGO [1, 2, 3] is at its initial target sensitivity, and the detection of an event from an astrophysical source could come at any time. Around the globe a world-wide network of detectors is emerging; VIRGO in Italy [4, [5], GEO in Germany [ [] ] and TAMA in Japan [7] are operating alongside the LIGO detectors in the U.S. in the quest for gravitational wave detection. In a few years advanced LIGO and advanced Virgo will come on-line, with a sensitivity increase of 10 over initial LIGO [8, 9, 10, 11], increasing the prospective detection rate significantly. It will be a great day for physics when gravitational waves are finally directly detected, but it will also be the birth of a new way of observing the universe and conducting astronomy and astrophysics.

The observations of expected sources should be dramatic: stellar collapse, pulsars, the inspiral of binary neutron stars followed by black hole formation, or even the stochastic background from the Big Bang. Gravitational wave astronomy will soon enter a regime where parameter estimation work will provide the means to make important astrophysical statements. Gravitational wave burst signals from rotating stellar core collapse and bounce, resulting in the formation of a proto-neutron star, are one of the more promising and potentially extremely valuable sources for ground based interferometric detectors. Gravitational wave burst events are typically characterized by their short duration (from a few milliseconds to about one second) and the absence of accurate theoret- ical predictions for their waveforms (as opposed to e.g. waveform templates for binary inspiral). If these events happen sufficiently close by, the ground based gravitational wave detectors will be able to observe them. Detection searches for gravitational wave bursts typically use methods that can identify unmodeled but short duration events [12]. LIGO [13, 14, 15], VIRGO [16], GEO [14], and TAMA [15, 17] have all recently conducted searches for gravitational wave bursts.

The prediction of the exact burst signal to be expected from a rotating stellar core collapse event depends on the complex interplay of general relativity, nuclear and particle physics. Furthermore, it is anticipated that the signal is produced by various emission mechanisms, first from the coherent motion of the collapsing and rebounding core during the proto-neutron star formation and then the ringing down of the nascent hot proto-neutron star (all in cases when the core rotates). This is then possibly followed by emission due to prompt convective motion behind the hydrodynamic shock in the central part of the star due to non-axisymmetric rotational instabilities for rapid rotation, as well as due to pulsations of the proto-neutron star, for instance, triggered by fall-back of matter onto it (see e.g. [18] for a comprehensive review). Only recently, the full complexity of the prospective emission mechanisms for gravitational waves in a stellar core collapse has become appreciated. Previously, research mainly concentrated on the signal contribution from the collapse, core bounce and ring-down phase, and therefore this is the gravitational wave signal that is now by far most comprehensively and accurately investigated us- 
ing numerical studies. Recently, a series of calculations of core collapse models with unprecedented physical realism has been performed 19, 20, 21]. These studies provide strong evidence that the predictions of gravitational wave signals from the collapse and bounce phase are now robust. The gravitational wave results from these investigations have been made publicly available in the form of electronic waveform catalogs. If detected, such a gravitational wave signal can then ideally provide information on the mass of the progenitor model, the precollapse rotation, the density of the collapsed core, and the nuclear equation of state.

However, even for the collapse and core bounce signal (not to mention the possible signal contributions from other emission mechanisms in a stellar core collapse event) one cannot conduct a template based search as is done when looking for coalescing compact binary signals, since it would be computationally impossible to completely cover the signal parameter space, and therefore various other methods have been developed to efficiently perform the search for gravitational wave burst signals in the detectors. Bayesian inferential methods provide a means to use data from interferometric gravitational wave detectors in order to extract information on the physical parameters associated with an event [22]. Markov chain Monte Carlo (MCMC) methods are a powerful computation technique for parameter estimation within this framework; they are especially useful in applications where the number of parameters is large 23]. Good descriptions of the positive aspects of a Bayesian analysis of scientific and astrophysical data are provided in [22, 24, 25]. MCMC routines have been developed that will produce parameter estimates for gravitational wave signals from binary inspiral [26, 27, 28, 29, 30, 31] and pulsars [32, 33, 34]. Accurate parameter estimation of observed gravitational radiation events is the necessary pathway to understanding important problems in astrophysics and cosmology. In this paper we present methods which are the starting point in the process of using MCMC methods to extract and estimate parameters associated with rotating stellar core collapse events from their gravitational wave signals emitted during the collapse, core bounce and ring-down phase.

Unfortunately, due to the complexity of the event (in particular its intrinsic multi-dimensional nature), the computational time required to derive these signals in numerical simulations of rotating stellar core collapse is significant, and thus the waveform generation cannot be performed instantly while fitting a signal template to the data, and additional techniques to simplify the analysis are required. Instead of using waveforms corresponding to arbitrarily picked locations within parameter space, we reduce the complexity of the problem by simplifying the waveform space to the span of a small number of basis vectors. The basis vectors are derived from a representative catalog of numerically computed signal waveforms through the use of principal component analysis (PCA) [35]. The waveforms used in our present analysis are from the most recent, advanced, and comprehensive general relativistic study of rotating stellar core collapse by Dimmelmeier et al. 21] and depend on the mass of the progenitor model, the precollapse rotation, and the nuclear equation of state.

In this study we make use of a catalog that is smaller than eventually required in an extensive and accurate analysis of signals in real data, but with its non-trivial number of elements spanning a large portion of the interesting parameter space we expect it nevertheless to be sufficiently complex to probe and to demonstrate the method qualitatively. The MCMC algorithm essentially fits a superposition of derived basis vectors to the data, providing parameter estimates as well as associated uncertainties. We also demonstrate how PCA and MCMC then allow the reconstruction of the time series of a gravitational wave burst signal, including confidence bands. We present initial results showing that these PCA basis vectors are actually physically meaningful, and that we can extract astrophysical parameters from the measured core collapse burst signal. The ultimate goal is to be able to extract information on the nuclear equation of state from the gravitational wave signal as it is observed by a network of interferometers, as well as other relevant physical parameters associated with the event, as far as this is possible from the collapse, bounce and ringdown signal alone.

There have been other approaches to signal reconstruction and parameter estimation with burst signals from stellar core collapse. The discipline essentially starts with the work of Gürsel and Tinto [36], who presented a method for reconstructing the time series for gravitational wave bursts. Rakhmanov 37] developed a general scheme of Tikhonov regularization to be applied on a network of detectors in order to extract the signal. MCMC methods have also been applied to parameter estimation for burst events from cosmic string cusps [38]. Searle et al. [39] have recently worked out the Bayesian framework to the coherent detection and analysis of burst signals, including a wide range of special cases like white noise burst signals, but also signals constituting linear combinations of some set of basis vectors, like the ones used in this present study. Summerscales et al. [40] proposed a maximum entropy technique in order to infer the timedependent gravitational wave burst signals detected by a network of interferometric detectors; they then calculated the correlation between their reconstructed waveform and the entries in a catalog of rotating core collapse waveforms [41] in order to infer the physical parameters from the event. Our work is different in that we use waveforms from physical models (the table of rotating core collapse signals) as our means of reconstructing the signal. PCA basis vectors are initially created from the table of waveforms, and the MCMC estimate of the amplitudes for the PCA basis vectors provides the reconstructed waveform. A linearly polarized gravitational wave signal can be reconstructed from the data of a single interferometer. The estimate of the physical parameters 
comes from an association of the reconstructed waveform with the elements of the catalog. The results in this paper are derived only using data from a single detector (signals embedded in simulated noise matching that of LIGO at its target sensitivity).

The organization of the paper is as follows. In Section [I] we describe the methods by which LIGO, VIRGO, GEO, and TAMA are searching for burst events; the parameter estimation technique we describe in this paper would be applied to event candidates from such a burst search. The method by which the catalog of stellar core collapse burst signals is established is described in Section III. A summary of the PCA method used in our study is presented in Section IV, while our analysis strategy is described in Section $\mathrm{V}$, We illustrate how the analysis method works when applied to simulated example data in Section VI, and in Section VII we demonstrate that the PC basis vectors and the inferred corresponding coefficients actually carry physically meaningful information. In Section VIII we provide a summary, and discuss the direction of future work.

\section{BURST SEARCH PIPELINES}

All collaborations associated with ground based interferometric gravitational wave detectors have developed burst search pipelines in order to look for events 13, 14, 15, 16, 17]. Although some gravitational wave burst sources are well modeled, the burst pipelines search for very general types of waveforms. The only assumption which is usually made concerns the duration of the signal (less than a few hundred of ms) and the frequency bandwidth of the search, which can be as large as the detector bandwidth (from a few dozen of $\mathrm{Hz}$ up to the Nyquist frequency). Burst pipelines typically reduce the frequency bandwidth to a few $\mathrm{kHz}$ to focus on specific types of sources.

Most of the gravitational wave burst pipelines look for an excess of energy in a time-frequency map using different multi-resolution time-frequency transforms. Anderson et al. [42] initially considered the energy given by the Fourier transform in a frequency band. More recent methods [43] make use of a wavelet decomposition of the data stream. In contrast, in [44, 45, 46] a gravitational wave burst signal represented as a sine-Gaussian is implemeted.

Usually the gravitational wave burst searches are performed using data from a network of detectors. Demanding that a gravitational wave burst event is seen simultaneously in several detectors allows one to reduce the false alarm rate, which is rather high in a burst search due to the short duration of the signal. This is also the only way to disentangle a real gravitational wave signal from transient noise events. There exist two kinds of network analysis: coincident or coherent filtering. In a coincidence analysis [44, 46], each interferometer output is analyzed, providing a list of triggers. A coinci- dence in time and frequency is then required. A coherent analysis 36, 47, 48, 49, 50, 51, 52] uses all interferometers' information by combining the input data streams or the filtered data streams into one single output which takes into account the detectors' antenna response function assuming the source is at a given position in the sky. A coherent analysis more efficienctly suppresses nonstationarities that are expected to be incoherent in the different detectors.

When using data from a network of detectors, burst pipelines can reconstruct the position in the sky of the source [36, 53, 54, 55]. The accuracy of the source sky position depends on the time resolution of the pipeline. It is usually estimated for a set of different waveforms. Depending on the complexity of the signal waveform the time resolution can vary from a fraction of ms up to several ms [12]. The frequency content (central frequency and bandwidth) of the event is also estimated by the burst pipelines. The frequency information can give some hints concerning the possible astrophysical source. For instance, a central frequency of a few hundred $\mathrm{Hz}$ would point towards the event of a core collapse in a massive star resulting in the formation of a proto-neutron star 21] (in particular if the detection is accompanied by a neutrino trigger following shortly after) while a higher frequency content at about $10 \mathrm{kHz}$ could suggest that the event could be due to a neutron star collapsing to a black hole [56]. Besides, coherent pipelines can also extract from the data an estimation of the waveform without assuming any model for the triggers with sufficient amplitude [36, 57].

The work presented in this paper intends to extract even more information from the most significant events found by a gravitational wave burst pipeline assuming an astrophysical source model. The estimation of the parameters could also be used to reject a possible gravitational wave event candidate; the idea of distinguishing a real stellar core collapse signal from an instrumental glitch is briefly discussed in sections VII and VIII below. The starting point for our technique would be the list of candidate triggers produced by a burst all-sky search pipeline, or times provided by electromagnetic and/or neutrino observations (external triggers). In both cases, the MCMC will search for the event over some relatively small time span. The trigger times provide a relatively small number of data periods to be examined by our method. The MCMC would attempt to produce a reconstruction of the signal based on a waveform catalog for stellar core collapse models.

\section{GRAVITATIONAL WAVE SIGNALS FROM ROTATING CORE COLLAPSE AND BOUNCE}

In stellar core collapse, the (possibly rotating) unstable iron core of a massive star at the end of its life contracts to supernuclear density on a dynamical time scale of the order of $100 \mathrm{~ms}$. As the core material stiffens due to re- 
pulsive nuclear forces, the collapse is halted abruptly, and the inner part of the core undergoes a rebound (the core bounce). After a period of ring-down oscillations, the hot proto-neutron star settles down, while the remainder of the star is possibly (mainly depending on the mass of the progenitor) blown off in a supernova explosion.

If the precollapse stellar core is rotating, the dynamics of the evolution are reflected in the gravitational wave signal waveform with a slow rise during the core collapse, a large negative peak around bounce, and damped oscillations in the ring-down phase. If rotational effects become important for rapidly rotating models, the core collapse can even be stopped by centrifugal forces alone at subnuclear density. However, in contrast to previous, less sophisticated studies of the stellar core collapse scenario (see e.g. 58] and references therein), Dimmelmeier et al. 20] have shown that the signal waveform remains qualitatively unaltered and is thus generic for a wide range of initial rotation strength.

The gravitational radiation waveforms analyzed in this article are the burst signals from the most recent, advanced, and comprehensive general relativistic study of rotating stellar core collapse models [21]. These simulations were performed with a computational code that utilizes accurate Riemann solvers to evolve the general relativistic hydrodynamic equations and a nonlinear elliptic solver based on spectral methods [59] for the fully coupled metric equations in the conformal flatness approximation of general relativity [60]. The precollapse iron core models were taken from recent stellar evolutionary calculations by Heger et al. [61, 62], either with an intrinsic or an artificially added rotation profile. These initial models were then evolved with a nonzero-temperature nuclear equation of state (EoS), either the one by Shen et al. 63, 64 (Shen EoS) or the one by Lattimer and Swesty [65, 66] (LS EoS; with a bulk incompressibility $K=180 \mathrm{MeV}$ ), both in the implementation of Marek et al. [67], including contributions from baryons, electrons, positrons, and photons. Deleptonization by electron capture on nuclei and free protons during the collapse phase is realized as proposed and tested by Liebendörfer [68].

Of the 136 models investigated in the study by Dimmelmeier et al. 21], 128 models have an analytic initial rotation profile. Their collapse behavior is determined by the following parameters:

- The strength of rotation is specified by the precollapse central angular velocity, which varies from $\Omega_{\mathrm{c}, \mathrm{i}}=0.45$ to $13.31 \mathrm{rad} \mathrm{s}^{-1}$ (with the individual range depending on the differentiality of rotation). The influence of rotation strength on the collapse dynamics and on the burst signal via rotational flattening is very pronounced.

For a wide range of slow to intermediate initial rotation strengths, the peak $|h|_{\text {max }}$ of the gravitational wave amplitude is almost proportional to the ratio $T_{\mathrm{b}} /|W|_{\mathrm{b}}$ of rotational energy to gravitational energy at bounce (which in turn increases approx- imately linear with $\Omega_{\mathrm{c}, \mathrm{i}}$ in this regime if all other model parameters are kept constant).

For very rapid rotation, however, $|h|_{\max }$ reaches a maximum and declines again, when the centrifugal barrier slows down the contraction considerably, preventing the core from collapsing to high supernuclear density. In such a case the frequency of the burst signal, which is practically constant for slow or moderate rotation, decreases significantly. Note that the trend to lower frequencies for very slowly rotating models shown in [20, 21], which spoils the constancy of the signal frequency for such models, is due to a (possibly artificial) low-frequency contribution originating from post-bounce convection in the proto-neutron star, which superimposes the signal from the core bounce and proto-neutron star ring-down.

- The differentiality of the precollapse rotation profile is set by a length scale with values $A=50000$, 1000 , or $500 \mathrm{~km}$, ranging from almost uniform to strongly differential rotation. For a fixed initial strength of rotation (as set by $\Omega_{\mathrm{c}, \mathrm{i}}$ ), a change in $A$ alone has no strong effect on the gravitational wave signal, neither on the amplitude nor on the frequency. However, rapid rotation can only be achieved for comparably small values of $A$.

- The set of precollapse cores encompasses models with a progenitor mass of $M_{\text {prog }}=11.2,15.0$, 20.0 , or $40.0 \mathrm{M}_{\odot}$ (masses at zero-age main sequence). The choice of the progenitor mass has a direct impact on the mass of the inner core during the collapse and at bounce (and thus on the mass of the proto-neutron star). Already without rotation, the different progenitors produce an inner core at bounce with a mass that depends non-monotonously on the mass of the progenitor (with the inner core mass increasing in the order $M_{\text {prog }}=11.2,20.0,15.0$, and $40.0 \mathrm{M}_{\odot}$ ).

This variation is considerably amplified by rotation, which itself increases the mass on the inner core at bounce (approximately linear with $\Omega_{\mathrm{c}, \mathrm{i}}$ at slow rotation and roughly quadratically at rapid rotation; see [21]). Nevertheless, the reflection of that effect on the peak signal amplitude $|h|_{\max }$ is practically negligible, as the effect of a high inner core mass (which causes a large quadrupole moment and thus a strong resulting gravitational wave signal) is canceled by strong centrifugal support, resulting in slower collapse dynamics and, consequently, a weaker gravitational wave signal.

The main frequency of the gravitational wave burst signal is also not influenced significantly by the mass of the progenitor model, except that in models with the least massive progenitor $\left(M_{\text {prog }}=\right.$ $11.2 \mathrm{M}_{\odot}$ ) even strong initial rotation does not decelerate the relatively small collapsing inner core 
enough to make it bounce at subnuclear densities. Hence the signal frequencies of such models remain comparably high. We also point out that the approximate nature of the deleptonization scheme during the collapse phase up to core bounce employed in these models could actually be responsible for overemphasizing the variation in the mass of the inner core at bounce with respect to the mass of the progenitor.

- The microphysical equation of state during the evolution is chosen to be either the Shen EoS or the LS EoS. In the subnuclear density regime, the two EoSs are rather similar, with the LS EoS being a bit softer on average. However, at supernuclear density, the differences are more pronounced. Here the adiabatic index $\gamma$ of the LS EoS jumps to $\sim 2.5$, while in the Shen $\operatorname{EoS} \gamma$ reaches values of $\sim 3.0$, making the nuclear material described by that EoS significantly stiffer. Consequently, the models with the Shen EoS consistently exhibit lower central densities at bounce and after ring-down.

Still, with respect to the peak waveform amplitude $|h|_{\max }$ of the burst signal, this does not translate into an unequivocal trend due to a complicated interplay in the proto-neutron star between central compactness and density structure at intermediate radii. The peak frequency of the waveform spectrum, on the other hand, is almost always lower if the Shen EoS is used, which is a direct consequence of the lower central densities in the bounce and post-bounce period due to the nuclear material stiffness from that EoS if all other parameters are identical and only the EoS is varied.

In Fig. 1 we present a representative sample of waveforms which illustrate the impact of a model's initial rotation state on the gravitational wave signal from core bounce, from slow and almost uniform initial rotation (model "s20a1005_shen"), moderately fast and modestly differential initial rotation (model "s20a2o09_shen"), to rapid and very differential initial rotation (model "s20a3o15_shen"). While the effect of varying the initial rotation state is clearly reflected in the waveform for the models shown here, the influence of the progenitor model's mass and also the EoS for a fixed initial rotation state is much less apparent, and thus analogous pictorial waveform comparisons are not presented.

We note that 8 progenitor models of [21] are from a stellar evolutionary calculation that includes rotation in an approximate way. Hence, these models already have an initial angular velocity profile; no artificial rotation according to the simple analytic relation, like in the other models, is added prior to evolution. Consequently, as initial rotation is not parametrized, these models are not of use for this study. However, the collapse dynamics and associated signal waveforms of these models are well represented by models with artificially added precollapse rotation in terms of both signal amplitude and frequency,

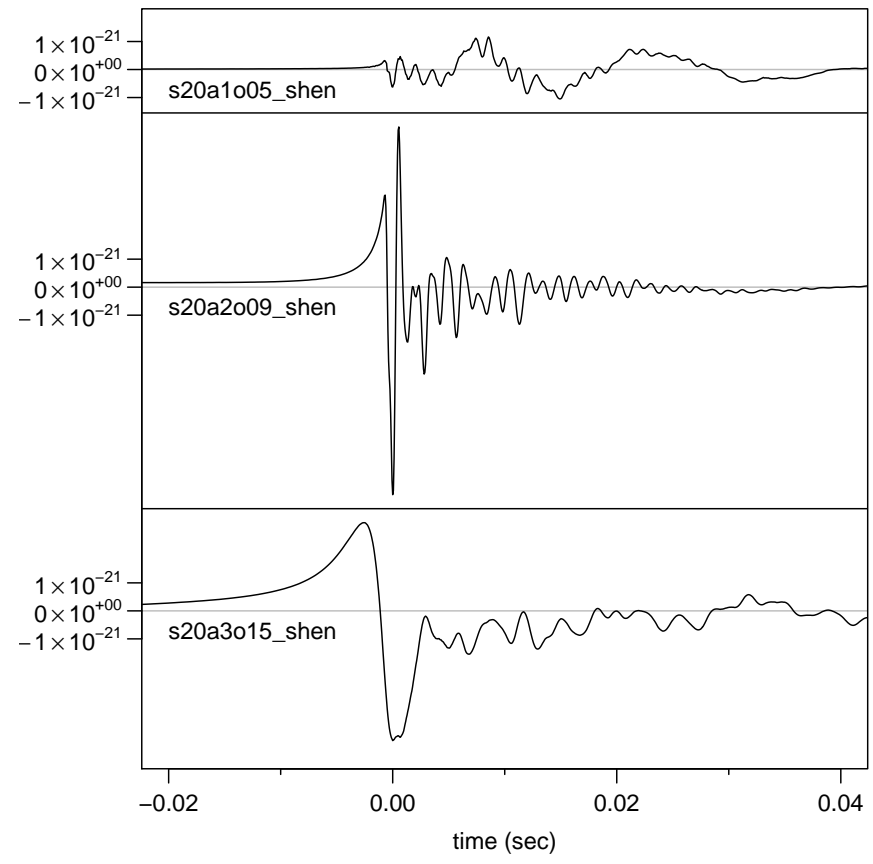

FIG. 1: Sample waveforms of the core bounce signal for three models with varying initial rotation states while the mass of the progenitor model and EoS are fixed. Note the relatively small signal peak at the time of core bounce and the significant late-time contribution from post-bounce convection for the slowly rotating model "s20a1o05_shen", and the overall lower signal frequency for the rotation-dominated and centrifugally bouncing model "s20a3o15_shen". The three signals presented here adequately cover the waveform morphology of our signal catalog.

and therefore it is justified to not separately consider their behavior here. The different influences of the various model parameters, which are summarized here only briefly, are discussed in detail in 21]. The respective signal data can be downloaded freely from an online waveform template catalog [69].

We emphasize that the parameter selection for the models in [21] is fairly complete in that it accounts for all known relevant parameters which could have an impact on the burst signal from a core bounce (although, for instance in the case of neutrino effect, in an approximate way). Furthermore, the astrophysically meaningful range of the parameters has been reasonably exhausted (in the case of rotation, the expected strength of rotation for the bulk of stellar core collapse events is even at the lower end of the investigated range; see [70]). Only the selection of EoS is limited to two due to a lack of access to alternative nonzero-temperature nuclear equations of state for stellar core collapse at the time when the study by Dimmelmeier et al. 21] was performed. However, with the Shen EoS and the LS EoS the two extremes of a rather stiff nuclear material and a somewhat soft one are probably well covered.

The range of frequencies and amplitudes of the signals is quite broad for the model sample of [21], with the inte- 
grated characteristic frequency $f_{\mathrm{c}}$ spanning from about 100 to $700 \mathrm{~Hz}$ (as opposed to the peak frequency $f_{\max }$ of the spectrum which lies in a narrow interval around $700 \mathrm{~Hz}$; see Fig. 16 in 21]) and the integrated dimensionless characteristic amplitude $h_{\mathrm{c}}$ ranging from $6 \times 10^{-22}$ to $7 \times 10^{-21}$ for e.g. initial LIGO and a distance to the source of $10 \mathrm{kpc}$ (for the definition of these quantities, see the references in e.g. 21]).

Still, the main factor of influence causing this variation is the rotation of the core (as measured by $\Omega_{\mathrm{c}, \mathrm{i}}$ and $A$, and rather well resolved in the models of [21]), while the mass of the progenitor, precollapse differentiality of rotation, and microphysical EoS hardly affect both $f_{\mathrm{c}}$ and $h_{\mathrm{c}}$ (see Fig. 17 in [21]). This partial degeneracy regarding the model parameters makes it very difficult to infer the unknown parameters from a detection of the gravitational wave burst signal emitted by a rotating stellar core collapse. In reverse, this means that parameters of little impact on the gravitational wave signal may be resolved only coarsely in a parameter study aiming at providing signal templates.

For an event with at most moderate rotation, in practice only the strength of rotation can be extracted from the waveform with confidence, provided the distance to the source and the orientation with respect to the rotation axis can also be determined. Only if a multitude of core collapse events can be detected via gravitational waves can systematic effects of e.g. the nuclear material stiffness (as described by the EoS) on the frequency also be analyzed with more certainty.

While the independent parameters $\Omega_{\mathrm{c}, \mathrm{i}}, A, M_{\mathrm{prog}}$, and EoS uniquely specify each collapse model, from the actual numerical simulation of the collapse a number of important quantities can be obtained which characterize the evolution of each individual model; these are typically presented as results for rotating stellar core collapse calculations. Among these are the maximum density $\rho_{\max , \mathrm{b}}$ at the time of core bounce, the ratio $T_{\mathrm{b}} /|W|_{\mathrm{b}}$ of rotational energy to gravitational energy at bounce and the corresponding value $T_{\mathrm{pb}} /|W|_{\mathrm{pb}}$ late after bounce.

These quantities provide information about the collapse dynamics and, for instance, permit one to distinguish between a core bounce that is mostly caused by the nuclear material stiffening at supernuclear densities to one that is dominated by centrifugal forces, which is again reflected by the waveform. Thus, in reverse the waveform encodes not only information about the independent model parameters, but also about these evolution quantities. Consequently, in the analysis on the correspondence between PCs and physical parameters presented in Section VII, we not only consider the parameters for the model setup but also the evolution of the quantities $\rho_{\max , \mathrm{b}}, T_{\mathrm{b}} /|W|_{\mathrm{b}}$, and $T_{\mathrm{pb}} /|W|_{\mathrm{pb}}$. However, in contrast to these 'robust' parameters reflecting the global collapse dynamics we refrain from analyzing other quantities (like e.g. entropy at a specific off-center location or the time span of contraction) which are prone to depend more sensitively on the numerical evolution scheme or grid setup used for the model simulation.

\section{SINGULAR VALUE DECOMPOSITION}

The signal waveforms used in the analysis reported here are originally generated at a higher sampling rate. Each waveform is subsequently resampled at a rate of $16384 \mathrm{~Hz}$ (the LIGO and GEO data sampling rate). The waveforms are then buffered with zeros so that they are of the same length. Finally, the waveforms are time shifted so that the first (negative) peak in each waveform (which occurs shortly after the time of core bounce) are aligned.

For modeling purposes, the set of signal waveforms will be decomposed into an orthonormal basis. Following the method prescribed by [35], we create a matrix $\mathbf{H}$ so that each column corresponds to a signal waveform from the catalog after subtracting the overall mean of the waveforms. For $m$ waveforms, each $n$ samples long, $\mathbf{H}$ is a matrix with dimensions $n \times m$. Using singular value decomposition [71], $\mathbf{H}$ is factorized such that

$$
\mathbf{H}=\mathbf{U S ~ V}^{\prime},
$$

where $\mathbf{U}$ and $\mathbf{V}$ are orthonormal $n \times r$ and $m \times r$ matrices, respectively, and $\mathbf{S}$ is a diagonal $r \times r$ matrix containing the singular values of $\mathbf{H}$ in decreasing order, i.e. $\mathbf{S}=\operatorname{diag}\left(s_{1}, \ldots, s_{r}\right)$ with $s_{1} \geq \ldots \geq s_{r}>0$, $r=\operatorname{rank}(\mathbf{H}) \leq \min (m, n)$. The columns of $\mathbf{U}$ are the eigenvectors of the empirical covariance matrix $\mathbf{H} \mathbf{H}^{\prime}$, and similarly, the columns of $\mathbf{V}$ are the eigenvectors of $\mathbf{H}^{\prime} \mathbf{H}$. Additionally, the singular values in $\mathbf{S}$ are the square roots of the eigenvalues $\lambda_{i}$ of either $\mathbf{H}^{\prime}$ or $\mathbf{H}^{\prime} \mathbf{H}$, i.e. $s_{i}=\sqrt{\lambda_{i}}$.

The columns of $\mathbf{U}$, i.e. $\boldsymbol{u}_{1}, \ldots, \boldsymbol{u}_{r}$, form an orthonormal basis of the linear space spanned by the columns of $\mathbf{H}$, i.e. the signal waveform space, and each signal waveform can now be uniquely represented as a linear combination of these eigenvectors. A measure of multivariate scatter about the mean is the trace of the empirical covariance matrix, $\operatorname{tr}\left(\mathbf{H} \mathbf{H}^{\prime}\right)$, also called total variation, which equals $\sum_{i}^{r} \lambda_{i}$. So the sum of the first $k \leq r$ largest eigenvalues, $\sum_{i=1}^{k} \lambda_{i}$, measures how much of the total variability of the waveforms is explained by the first $k$ eigenvectors. These are referred to as the first $k$ "principal components" (PCs) and achieve an optimal dimension reduction from the $r$-dimensional signal waveform space to a $k$-dimensional subspace.

Note that each waveform is $n$ samples long and $\mathbf{U}$ has dimensions $n \times r$. For the waveforms considered in this article, $n$ is typically 1000 to 10000 samples long, so computing the eigenvectors of $\mathbf{H} \mathbf{H}^{\prime}$ in $\mathbf{U}$ can be computationally expensive. On the other hand, the number $m$ of waveforms in the catalog is of the order of 100 . So, computing the eigenvectors of $\mathbf{H}^{\prime} \mathbf{H}$ in $\mathbf{V}$ is much less demanding, and they can be used to compute the eigenvectors in $\mathbf{U}$ by first noting that

$$
\mathbf{H}^{\prime} \mathbf{H} \boldsymbol{v}_{i}=\lambda_{i} \boldsymbol{v}_{i}
$$




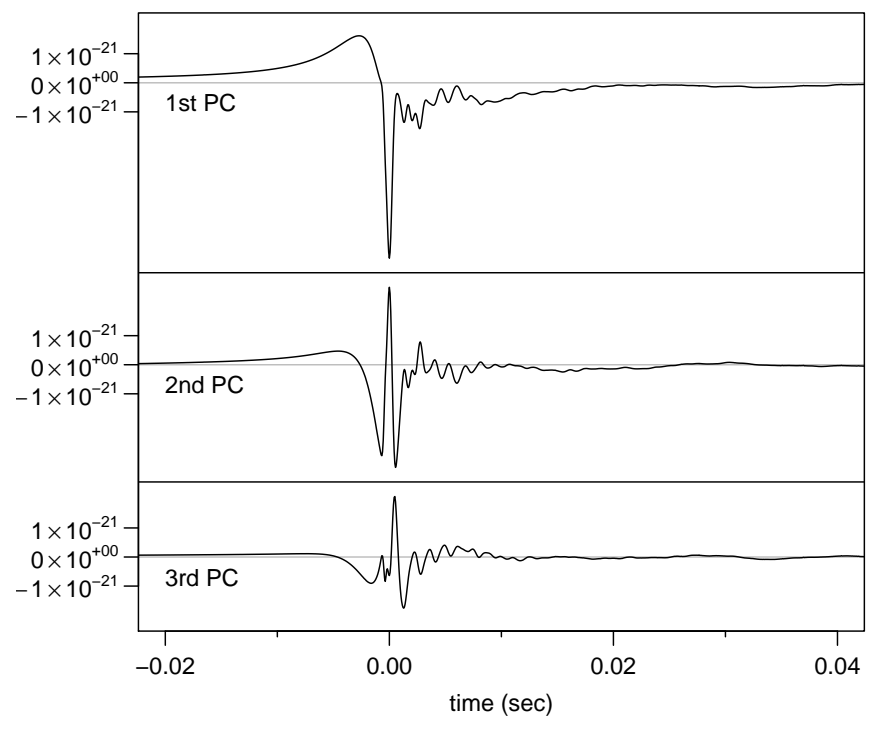

FIG. 2: The top three principal components (PCs) derived from the catalog of waveforms described in Sec. III

where $\boldsymbol{v}_{i}$ is the eigenvector of $\mathbf{H}^{\prime} \mathbf{H}$ corresponding to the $i$ th largest eigenvalue $\lambda_{i}$. By pre-multiplying both sides with $\mathbf{H}$, we obtain

$$
\mathbf{H} \mathbf{H}^{\prime} \mathbf{H} \boldsymbol{v}_{i}=\lambda_{i} \mathbf{H} \boldsymbol{v}_{i} .
$$

From this, we can see that $\mathbf{H} \boldsymbol{v}_{i}=\boldsymbol{u}_{i}$, i.e. $\mathbf{H} \boldsymbol{v}_{i}$ is the eigenvector corresponding to the $i$ th largest eigenvalue of $\mathbf{H} \mathbf{H}^{\prime}$ and thus equals the $i$ th column of $\mathbf{U}$. Fig. 2 illustrates the resulting first three PCs when applying this procedure to an actual waveform catalog.

\section{ANALYSIS STRATEGY}

\section{A. Definitions}

The starting point of our analysis is a waveform cata$\log$, i.e. a catalog of time series (of equal lengths) describing the gravitational wave signal of core collapse events corresponding to different input parameter settings. Let $n$ denote the number of samples (discrete time points) in each waveform vector and $m$ the number of waveform vectors in the catalog. As detailed in Section [V] for these $m$ time series we derive the first $k<r$ eigenvectors, $\boldsymbol{x}_{1}, \ldots, \boldsymbol{x}_{k}$, corresponding to the $k$ largest eigenvalues 35]. Each of these eigenvectors again is of length $n$.

The data to be analyzed (the noisy measurement) are given in the form of a time series vector $\boldsymbol{y}$ of length $N(N>n)$. This vector consists of additive nonwhite Gaussian noise with known (one-sided) power spectral density denoted by $S_{1}(f)$, superimposed by a corebounce burst signal of length $n$ located at an unknown instant $T$ along the time axis.

Our aim is to model the core-bounce burst signal observation in terms of the basis of the $k$ eigenvectors de- scribed above. To this end, we assume that the mean of the $y_{i}$ 's is a linear combination $\beta_{1} x_{i, 1}+\ldots+\beta_{k} x_{i, k}$ of the $k$ eigenvectors, and zero before and after the burst signal. In matrix notation, this can be expressed in terms of the expectation value $\mathrm{E}[\boldsymbol{y}]=\boldsymbol{X}_{(T)} \boldsymbol{\beta}$, where $\boldsymbol{\beta}=\left(\beta_{1}, \ldots, \beta_{k}\right)^{\prime}$ denotes the vector of regression coefficients, and the $N \times k$ matrix $\boldsymbol{X}_{(T)}$ has column vectors formed by the zeropadded $k$ largest eigenvectors $\boldsymbol{x}_{1}, \ldots, \boldsymbol{x}_{k}$ which are cyclically time-shifted by a lag $T$.

The signal reconstruction is eventually accomplished based on the Fourier domain representations of data and signal; in the following we will be referring to the conventions explicated in Appendix $\mathrm{A}$. Let $\tilde{\boldsymbol{y}}$ denote the Fourier transformed data vector and $\tilde{\boldsymbol{x}}_{i}, i=1, \ldots, k$, denote the discretely Fourier transformed eigenvectors after zero-padding each to length $N$. The real and imaginary parts of these form the columns of the $N \times k$ real-valued matrix $\tilde{\boldsymbol{X}}$ (neglecting the redundant elements due to hermitian symmetry).

One of the unknown parameters to be estimated is the signal's location $T$ along the time axis, and in order to match data and signal, one needs to be able to shift both against each other in time. Let $\tilde{\boldsymbol{X}}_{(T)}$ be the matrix of Fourier domain basis vectors shifted in time so that these correspond to a particular signal arrival time $T$ (with respect to some pivotal time point). Time-shifting of $\tilde{\boldsymbol{X}}$ by some lag $T$ can be done directly in the frequency domain by multiplying the original Fourier transform by a factor of $\exp (-2 \pi \mathrm{i} f T)$.

\section{B. Model 1: Basic linear regression model}

If the data were an exact linear combination of first $k$ principal components plus measurement noise, then the following standard linear model would adequately model the situation:

$$
\boldsymbol{y}=\boldsymbol{X}_{(T)} \boldsymbol{\beta}+\boldsymbol{\varepsilon},
$$

where $\varepsilon$ is the (Gaussian) noise vector with given (onesided) spectral density $S_{1}(f)$. In the frequency domain this corresponds to

$$
\tilde{\boldsymbol{y}}=\tilde{\boldsymbol{X}}_{(T)} \boldsymbol{\beta}+\tilde{\boldsymbol{\varepsilon}},
$$

where $\tilde{\varepsilon}$ now is the Fourier transformed noise vector. In Fourier domain, the real and imaginary components of the noise vector $\tilde{\varepsilon}$ then simply are independently zeromean Gaussian distributed with variances proportional to the power spectral density $S_{1}(f)$ [72, 73] (see also Appendix B).

What is known here are the data (measurement) $\boldsymbol{y}$, the matrix $\boldsymbol{X}$ of basis vectors (derived from the waveform catalog through PCA), and the noise's power spectral density. The unknowns are the coefficients $\boldsymbol{\beta}$ and the time parameter $T$. The a priori information about these is expressed in the prior distribution $\mathrm{P}(\boldsymbol{\beta}, T)$, which is assumed to be uniform, i.e. any value is assumed equally likely. 


\section{Model 2: Random effects regression model}

In general, the basis vectors will not allow one to completely reconstruct the original signal, there will always be a certain mismatch left unexplained when simplifying the problem to the reduced set of PCs as in (5) above. Neglecting the mismatch term would result in overconfidence in the reconstructed waveform and parameters. However, the simplified model introduced above will still be of interest, as it provides good approximations that are useful in the eventual implementation.

Adding an extra mismatch error component $\boldsymbol{m}$ to the model, a random effect in statistical terminology, the model is now

$$
\tilde{\boldsymbol{y}}=\tilde{\boldsymbol{X}}_{(T)} \boldsymbol{\beta}+\tilde{\boldsymbol{m}}+\tilde{\boldsymbol{\varepsilon}} .
$$

In the following, we expect the original catalog to be sufficiently densely populated, so that the mismatch is primarily due to the effect of neglecting PCs, and not due to the signal being much unlike the ones in the catalog. All we assume to be known about the mismatch is that it contributes a certain fractional amount to the signal's power. That amount was expressed in [35] in terms of the match parameter $\mu$. Taking the difference between reconstructed and actual signal to be zero on average, and then expecting a certain (fractional) power for the mismatch, we use a Gaussian distribution with corresponding mean and variance for modelling the mismatch,

$$
\mathrm{P}\left(m_{i} \mid \sigma_{m}^{2}\right)=\mathrm{N}\left(0, \sigma_{m}^{2}\right) \Leftrightarrow \mathrm{P}\left(\tilde{m}_{j} \mid \sigma_{m}^{2}\right)=\mathrm{N}\left(0, \frac{N}{2} \sigma_{m}^{2}\right),
$$

i.e. the mismatch $\boldsymbol{m}$ enters the model as an additional white noise component with a one-sided power spectral density of $2 \Delta_{t} \sigma_{m}^{2}$. The assumption of a Gaussian distribution is a simple and convenient choice here, and according to maximum entropy theory it also constitutes the most conservative possible choice 74]. It also seems to perform well in practice, despite the fact that the actual mismatch follows a rather heavy-tailed distribution with many near-zero and also a substantial number of extreme values. Since the mismatch is supposed to scale with the signal (i.e. the 'relative mismatch' is assumed constant), the mismatch's variance parameter $\sigma_{m}^{2}$ is set such that

$$
\sigma_{m}^{2} \approx \gamma^{2} \frac{1}{N}\|\boldsymbol{X} \boldsymbol{\beta}\|^{2}
$$

depending on the PC coefficients $\boldsymbol{\beta}$ via the implied sumof-squares (or power) of the PC contribution to the signal,

$$
\frac{1}{N}\|\boldsymbol{X} \boldsymbol{\beta}\|^{2}=\frac{1}{N} \sum_{i=1}^{N}\left(\sum_{j=1}^{Z} \beta_{j} x_{i, j}\right)^{2},
$$

and the scaling factor $\gamma^{2}=\frac{1-\mu^{2}}{\mu^{2}}$ is set so that it corresponds to a particular match $\mu$ as in 35. The above relationship between $\mu$ and $\gamma^{2}$ results from assuming the PC and mismatch contributions to the signal $\boldsymbol{s}=\boldsymbol{X} \boldsymbol{\beta}+\boldsymbol{m}$ to be (approximately) orthogonal: $\boldsymbol{m} \perp \boldsymbol{X} \boldsymbol{\beta}$ (i.e. the mismatch is defined as what is not spanned by the set of PCs).

The actual amount of mismatch is another unknown, and the (approximate) scaling of $\sigma_{m}^{2}$ with the signal power as in Eq. (8) is ensured through the definition of the prior distribution, which is set up as

$$
\mathrm{P}\left(\boldsymbol{\beta}, T, \sigma_{m}^{2}\right)=\mathrm{P}(T) \times \underbrace{\mathrm{P}\left(\boldsymbol{\beta}, \sigma_{m}^{2}\right)}_{=\mathrm{P}(\boldsymbol{\beta}) \times \mathrm{P}\left(\sigma_{m}^{2} \mid \boldsymbol{\beta}\right)} .
$$

As in the previous Section $\mathrm{VB}$, the priors $\mathrm{P}(T)$ and $\mathrm{P}(\boldsymbol{\beta})$ are set to be independent and uniform, but the conditional prior distribution $\mathrm{P}\left(\sigma_{m}^{2} \mid \boldsymbol{\beta}\right)$ is taken to be a scaled inverse $\chi^{2}$ distribution:

$$
\mathrm{P}_{\nu, \gamma^{2}}\left(\sigma_{m}^{2} \mid \boldsymbol{\beta}\right)=\operatorname{Inv}-\chi^{2}\left(\nu, \gamma^{2} \frac{1}{N}\|\boldsymbol{X} \boldsymbol{\beta}\|^{2}\right)
$$

with degrees-of-freedom parameter $\nu$ and scale parameter $\left(\gamma^{2} \frac{1}{N}\|\boldsymbol{X} \boldsymbol{\beta}\|^{2}\right)$, so that the prior certainty in the scale of $\sigma_{m}^{2} \mid \boldsymbol{\beta}$ is defined through $\nu$. For example, a specification of $\gamma^{2}=0.1$ and $\nu=3$ implies for the prior that $\mathrm{P}\left(0.038<\sigma^{2} /\left(\frac{1}{N}\|\boldsymbol{X} \boldsymbol{\beta}\|^{2}\right)<0.85\right)=90 \%=$ $\mathrm{P}(0.73<\mu<0.98)$, and a conditional prior mean of $\mathrm{E}\left[\sigma_{m}^{2} \mid \boldsymbol{\beta}\right]=\frac{\nu}{\nu-2} \gamma^{2} \frac{1}{N}\|\boldsymbol{X} \boldsymbol{\beta}\|^{2}$. Setting $\nu=0$ yields the (improper) Jeffreys prior, which does not depend on its prior scale parameter [75]. The Inv- $\chi^{2}$ distribution was chosen here because it constitutes the conjugate prior distribution for this problem [75], which makes it a 'natural' choice and makes the eventual implementation particularly simple, as will be seen in Section VD below. The parameters $\gamma^{2}$ and $\nu$ may now be set so that the prior distribution reflects the reconstruction accuracy to be expected from the given set of $k$ basis vectors derived from the waveform catalog at hand.

\section{Monte Carlo integration}

Inference on waveforms and parameters usually requires integrating the parameters' posterior distribution, as one is interested in figures like posterior expectations, quantiles, or marginal distributions. These are here determined using stochastic (Monte Carlo) integration, i.e. by generating samples from the posterior probability distribution and then approximating the desired integrals by sample statistics (means by averages, etc). The generation of samples from the posterior distribution is done using Markov chain Monte Carlo (MCMC) methods, that is, by designing a Markov process whose stationary distribution is the posterior probability distribution of interest, and which may then be numerically simulated step-bystep to produce the desired posterior samples. The generated samples then allow one to explore marginal or joint distributions of individual parameters, or of functions of the parameters as in the case of signal reconstruction, where the posterior distribution of $\tilde{\boldsymbol{X}}_{(T)} \boldsymbol{\beta}+\tilde{\boldsymbol{m}}$ is of interest, and $T, \boldsymbol{\beta}$ and $\tilde{\boldsymbol{m}}$ are random variables. 
In the case of the basic linear model (see Section VB), the set of unknown parameters consists of the vector of PC coefficients $\boldsymbol{\beta}$ and the time shift parameter $T$. An MCMC algorithm here may be implemented as a Gibbs sampler [75], since the conditional posterior distribution $\mathrm{P}(\boldsymbol{\beta} \mid T, \boldsymbol{y})$ of coefficients $\boldsymbol{\beta}$ for a given time $T$ is already known and easy to sample from (see Eq. (12) below). In a Gibbs sampler, generating samples from the joint distribution is carried out by alternately sampling from the two conditional distributions $\mathrm{P}(\boldsymbol{\beta} \mid T, \boldsymbol{y})$ and $\mathrm{P}(T \mid \boldsymbol{\beta}, \boldsymbol{y})$ 75]. Samples from $\mathrm{P}(\boldsymbol{\beta} \mid T, \boldsymbol{y})$ can be generated straight away, while a simple "Metropolis-withinGibbs" step, i.e. a nested Metropolis sampler implementation [75], may be utilized for sampling from from the (one-dimensional) distribution $\mathrm{P}(T \mid \boldsymbol{\beta}, \boldsymbol{y})$.

For the basic linear regression model (see Section VB), the conditional posterior distribution of the PC coefficients $\boldsymbol{\beta}$ for a given time shift $T$ is a multivariate Gaussian distribution:

$$
\mathrm{P}(\boldsymbol{\beta} \mid T, \boldsymbol{y})=\mathrm{N}\left(\hat{\boldsymbol{\mu}}_{T}, \hat{\boldsymbol{\Sigma}}_{T}\right),
$$

where

$$
\begin{aligned}
\hat{\boldsymbol{\Sigma}}_{T} & =\left(\tilde{\boldsymbol{X}}_{(T)}^{\prime} D^{-1} \tilde{\boldsymbol{X}}_{(T)}\right)^{-1}, \\
\hat{\boldsymbol{\mu}}_{T} & =\left(\tilde{\boldsymbol{X}}_{(T)}^{\prime} D^{-1} \tilde{\boldsymbol{X}}_{(T)}\right)^{-1} \tilde{\boldsymbol{X}}_{(T)}^{\prime} D^{-1} \tilde{\boldsymbol{y}},
\end{aligned}
$$

and $D=\operatorname{diag}\left(\sigma^{2}\left(f_{j}\right)\right)$ is the noise's covariance matrix, a diagonal matrix of the individual variances, as given in Eq. (B1) 75].

Sampling from the posterior distribution works similarly for the extended model (see Section VC), in addition to $\boldsymbol{\beta}$ and $T$, the mismatch $\tilde{\boldsymbol{m}}$ and mismatch variance $\sigma_{m}^{2}$ need to be sampled from. Note that the conditional posterior distribution $\mathrm{P}\left(\boldsymbol{\beta} \mid T, \tilde{\boldsymbol{m}}, \sigma_{m}^{2}, \boldsymbol{y}\right)$ is not simply multivariate Gaussian any more, since changes in $\boldsymbol{\beta}$ lead to different prior density values (via its effect on the signal's sum-of-squares value). The expression in Eq. (12) still is an excellent approximation and is useful for defining a proposal distribution within the Metropolis step of the algorithm. The conditional distribution of the mismatch vector $\tilde{\boldsymbol{m}}$ is independent Gaussian:

$$
\mathrm{P}\left(\tilde{\boldsymbol{m}} \mid \boldsymbol{\beta}, T, \sigma_{m}^{2}, \boldsymbol{y}\right)=\mathrm{N}(\check{\boldsymbol{\mu}}, \check{\boldsymbol{\Sigma}}),
$$

where

$$
\begin{aligned}
\check{\mu}_{j, \operatorname{Re}} & =\operatorname{Re}\left(\left(\tilde{\boldsymbol{y}}-\tilde{\boldsymbol{X}}_{(T)} \boldsymbol{\beta}\right)_{j}\right) \frac{\frac{N}{2} \sigma_{m}^{2}}{\frac{N}{2} \sigma_{m}^{2}+\frac{N}{4 \Delta_{t}} S_{1}\left(f_{j}\right)}, \\
\check{\sigma}_{j j, \operatorname{Re}}^{2} & =\frac{\frac{N}{2} \sigma_{m}^{2} \frac{N}{4 \Delta_{t}} S_{1}\left(f_{j}\right)}{\frac{N}{2} \sigma_{m}^{2}+\frac{N}{4 \Delta_{t}} S_{1}\left(f_{j}\right)},
\end{aligned}
$$

and analogously for the imaginary parts $\operatorname{Im}\left(\tilde{m}_{j}\right)$. For the mismatch variance $\sigma_{m}^{2}$ the conditional posterior is

$$
\mathrm{P}\left(\sigma_{m}^{2} \mid \boldsymbol{y}, \boldsymbol{\beta}, T, \tilde{\boldsymbol{m}}\right)=\operatorname{Inv}-\chi^{2}\left(\kappa, s^{2}\right),
$$

where the degrees of freedom $\kappa$ and scale $s^{2}$ are

$$
\begin{aligned}
\kappa & =\nu+N, \\
s^{2} & =\frac{\nu \gamma^{2} \frac{1}{N}\|\boldsymbol{X} \boldsymbol{\beta}\|^{2}+\sum_{i=0}^{N} m_{i}^{2}}{\nu+N}
\end{aligned}
$$

(see also Appendix C).

The MCMC sampler was eventually implemented as a Gibbs sampler, alternately sampling from the three conditional distributions of

$$
\begin{array}{ll}
\text { 1. } & \boldsymbol{\beta}, T \mid \boldsymbol{y}, \tilde{\boldsymbol{m}}, \sigma_{m}^{2}, \\
\text { 2. } & \tilde{\boldsymbol{m}} \mid \boldsymbol{y}, \boldsymbol{\beta}, T, \sigma_{m}^{2}, \text { and } \\
\text { 3. } & \sigma_{m}^{2} \mid \boldsymbol{y}, \boldsymbol{\beta}, T, \tilde{\boldsymbol{m}} .
\end{array}
$$

The first step is done in a "Metropolis-within-Gibbs" step, using a symmetric proposal distribution for $T$, the approximated posterior of $\beta \mid T, \ldots$ from Eq. (12) for the corresponding $\boldsymbol{\beta}$ proposal, and accepting/rejecting as in a usual Metropolis-Hastings sampler based on corresponding posterior and proposal probability density values. Samples for the second and third step may be generated directly.

\section{IMPLEMENTATION AND APPLICATION}

\section{A. Setup}

In the following examples we are using the waveform catalog described in Section [II] containing 128 gravitational radiation waveforms from rotating core collapse and bounce [21, 69]. The basis vectors to be used for signal reconstruction are generated through a PCA (see Section [V] [35]. Utilizing the same code and general setup for calculating the models described in [21, 69], we then compute three new rotating stellar core collapse models with input parameter values that did not appear in the original catalog (but lie within the range of the catalog parameter space) and their associated waveforms. The aim is then to reconstruct these waveforms using the PCA method.

The simulated data used here is $1 \mathrm{~s}$ in length and sampled at $16384 \mathrm{~Hz}$, superimposed with (simulated) nonwhite Gaussian noise, and Tukey-windowed. The shape of the noise curve is here taken to correspond to LIGO at its initial sensitivity as stated in [76]; this is the definition that is also implemented in the LIGO scientific collaboration's LSC algorithm library (LAL) [77]. The noise's spectral density $S_{1}(f)$ is estimated by averaging over a thousand "empirical" periodograms of identically generated data (same size and resolution, same windowing applied), more closely resembling a realistic case in which the noise spectrum would need to be estimated as well. In addition, this way convolution effects on the spectrum due to the finite data size and windowing are compensated.

The prior distribution for the mismatch parameter $\sigma_{m}^{2}$ is set by determining what match $\mu$ would be achievable for the given number $k$ of PCs. This is done by projecting each single waveform in the catalog onto the span of the PCs, and then (for any given number $k$ of PCs) considering the distribution of achieved mismatch values 


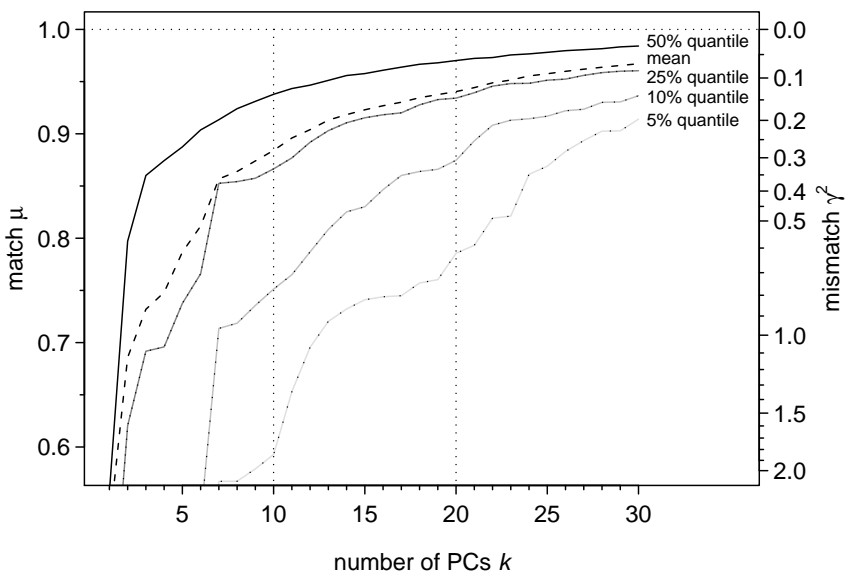

FIG. 3: Achievable match $\mu$ and scaling factor $\gamma^{2}$ for given numbers $k$ of PCs across the whole catalog used here. A particular number $k$ of $\mathrm{PC}$ basis vectors yields a certain match $\mu$ for each of the 128 waveforms in the catalog. Shown here is the distribution of matches across the catalog for increasing values of $k$, as characterised by mean, median and some more quantiles.

across the catalog. Fig. 3 illustrates the mean, median and some more quantiles of the mismatch distribution for increasing numbers of PCs (in analogy to Fig. 2 in Ref. [35]). We chose the prior's parameters (scale $\gamma^{2}$ and degrees-of-freedom $\nu$ ) so that it matched the distribution of computed matches across the catalog. For the two example settings of $k=10$ and $k=20$ considered in the following, this leads to maximum likelihood estimates of $\left(\gamma^{2}=13.3 \%, \nu=2.82\right)$ and $\left(\gamma^{2}=5.70 \%, \nu=2.96\right)$. The resulting prior density for $k=10 \mathrm{PCs}$ is also shown in Fig. 4 .

\section{B. Examples}

The first example illustrated here, labeled signal $A$ in the following, has a signal-to-noise ratio (SNR) of $\rho=10$, where the SNR is defined as

$$
\rho=\sqrt{4 \sum_{j} \frac{\frac{\Delta_{t}}{N}\left|\tilde{h}\left(f_{j}\right)\right|^{2}}{S_{1}\left(f_{j}\right)}} .
$$

It was generated assuming the Shen EoS, a mass $M_{\text {prog }}=$ $20 \mathrm{M}_{\odot}$ for the progenitor, a precollapse central angular velocity $\Omega_{\mathrm{c}, \mathrm{i}}=5.48 \mathrm{rad} \mathrm{s}^{-1}$, a differentiality of the precollapse rotation profile given by $A=1000 \mathrm{~km}$, and an effective distance of $d_{\mathrm{e}}=5.17 \mathrm{kpc}$. The effective distance depends on the actual distance to the source, and also reflects the effect on the amplitude of the gravitational wave signal from other parameters (such as the geometry of the detector with respect to the source), and is in general greater than the actual distance. The signal is embedded within simulated interferometer noise, and, using the model with $k=10$ basis vectors, the posterior distributions of the model parameters are derived using an MCMC implementation as described in the previous Section VD. The maximum achievable match for this example waveform (for $k=10$ and varying time shift $T$ ) is $\mu=0.97$.

The marginal posterior distributions of some individual parameters are illustrated in Fig. 4. Note the timing accuracy, which has a standard error of $0.1 \mathrm{~ms}$ in this case; in the following examples (for the same SNR), these are also of the order of sub-millisecond. The parameters' joint posterior distribution provides the posterior probability distribution of the waveform at any given time $t_{i}$. Fig. 5 shows the posterior mean and a $90 \%$ confidence band in comparison with the originally injected waveform.

The example signal $B$ illustrates how the signal recovery changes with differing numbers of PCs included in the model. This signal was generated assuming the $L S$ EoS, a mass $M_{\text {prog }}=15.0 \mathrm{M}_{\odot}$ for the progenitor, a precollapse central angular velocity $\Omega_{\mathrm{c}, \mathrm{i}}=2.825 \mathrm{rad} \mathrm{s}^{-1}$, a differentiality of the precollapse rotation profile of $A=1000 \mathrm{~km}$, and an effective distance of $d_{\mathrm{e}}=3.24 \mathrm{kpc}$. The signal's match is $\mu=0.89$ when using $10 \mathrm{PCs}$, and $\mu=0.95$ for 20 PCs. The same data are analyzed twice, once using $k=10$ and then using $k=20$ PCs; the resulting recovered waveforms are shown in Fig. 6. The enlarged model in principle allows for a better match of the signal, but on the other hand the larger number of parameters also decreases the certainty in parameter estimates, so that the recovery does not necessarily improve. In this example, the posterior variances of the $\mathrm{PC}$ parameters common to both models $\left(\beta_{1}, \ldots, \beta_{10}\right)$ as well as the time parameter $T$ increased for the $k=20$ case. In the resulting signal reconstruction (see Fig. 6) the confidence band is wider, and the discrepancy between injected signal and posterior mean also increases.

The reconstruction of signals at different SNRs can be seen in the following example signal $C$ in Fig. 7) here the same signal is injected at different overall amplitudes and hence SNRs $(\rho=10$ and $\rho=20)$. The injected signal was generated using the LS EoS, a mass $M_{\text {prog }}=40.0 \mathrm{M}_{\odot}$ for the progenitor, a precollapse central angular velocity $\Omega_{\mathrm{c}, \mathrm{i}}=9.596 \mathrm{rad} \mathrm{s}^{-1}$, a differentiality of the precollapse rotation profile given by $A=500 \mathrm{~km}$, and effective distances of $d_{\mathrm{e}}=8.32$ and $4.16 \mathrm{kpc}$, respectively. The resulting signal waveform's match is $\mu=0.97$. As expected, a higher SNR yields a more accurate recovery, and the resulting posterior variances are correspondingly smaller.

\section{CORRESPONDENCE BETWEEN PRINCIPAL COMPONENTS AND PHYSICAL PARAMETERS}

The analysis performed above not only allows one to reconstruct the waveform, but the posterior distribution of the PC coefficients actually also contains information 

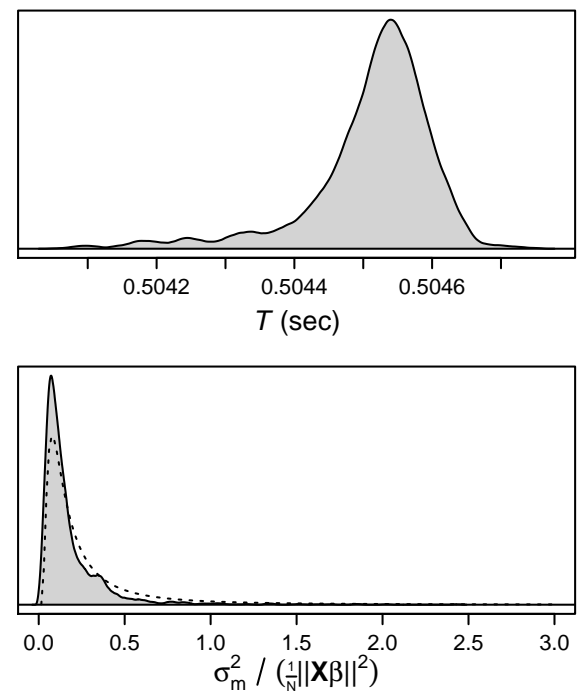
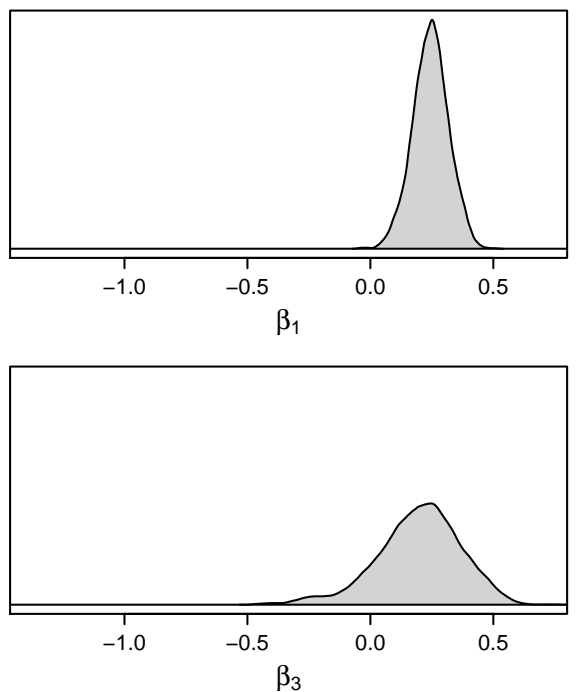
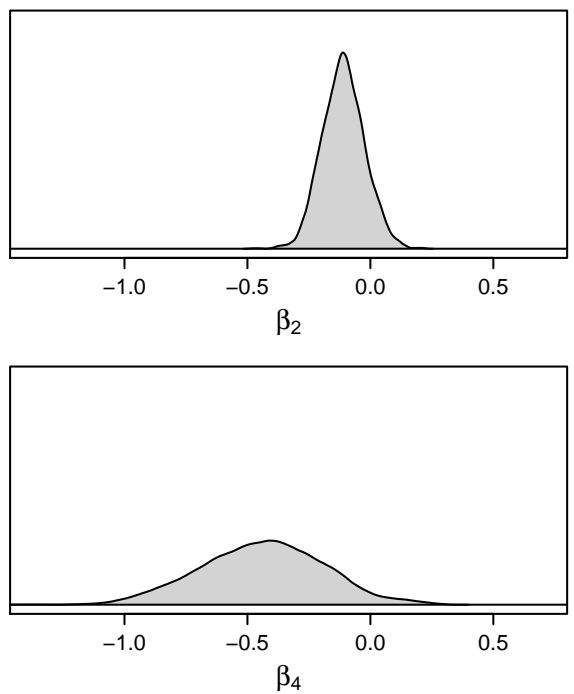

FIG. 4: Marginal posterior probability distributions of the parameters of example signal $A$ with SNR $\rho=10$, indicating the values and uncertainties of the model parameters as inferred from the data. Only the first four coefficients $\beta_{1}$ to $\beta_{4}$ corresponding to the top four principal components are shown here; there are $k=10$ coefficients in total. The dashed line in the top right plot shows the prior probability distribution in comparison to the (very similar) posterior.

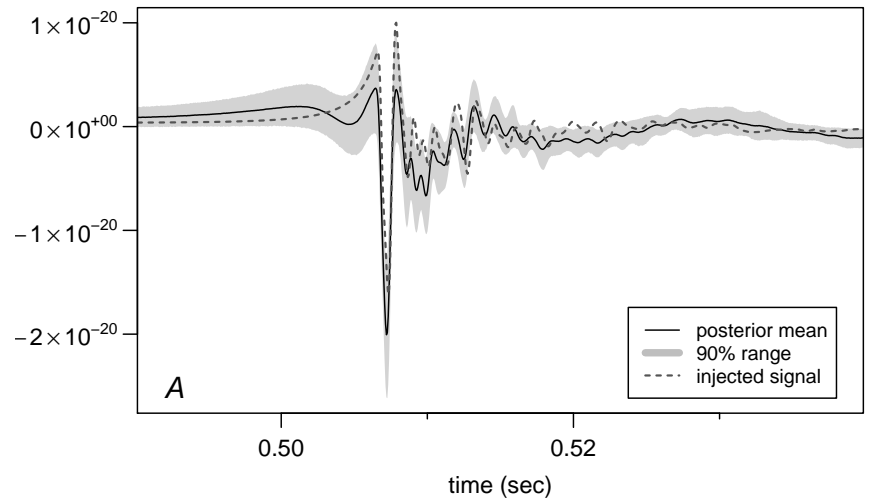

FIG. 5: Reconstruction of the example signal $A$ with SNR $\rho=10$ using $k=10$ PCs.

on the signal's physical parameters $\left(\Omega_{\mathrm{c}, \mathrm{i}}, A, M_{\mathrm{prog}}\right.$, and EoS) as well as other evolution parameters (like $\rho_{\max , \mathrm{b}}$, $T_{\mathrm{b}} /|W|_{\mathrm{b}}$, and $\left.T_{\mathrm{pb}} /|W|_{\mathrm{pb}}\right)$. In the present study we are only able to choose between two specific EoSs, but as this work progresses the goal is to make estimates from a wider selection of EoSs. Considering the catalog of waveforms alone, one can project each waveform onto the span of the PCs (as was done in the construction of Fig. 31) and inspect the resulting fitted PC coefficients. Fig. 8 illustrates the values of the first three coefficients $\left(\beta_{1}, \beta_{2}\right.$, and $\left.\beta_{3}\right)$ for all the signals in the catalog. Obviously the distribution of coefficients in parameter space exhibits some structure. This structure can now be exploited, the idea being that the posterior means and variances of the coefficients $\beta_{i}$ for a measured signal expose a pattern that is characteristic for the type of signal. For illustration, in the following we describe a simple approach involving the

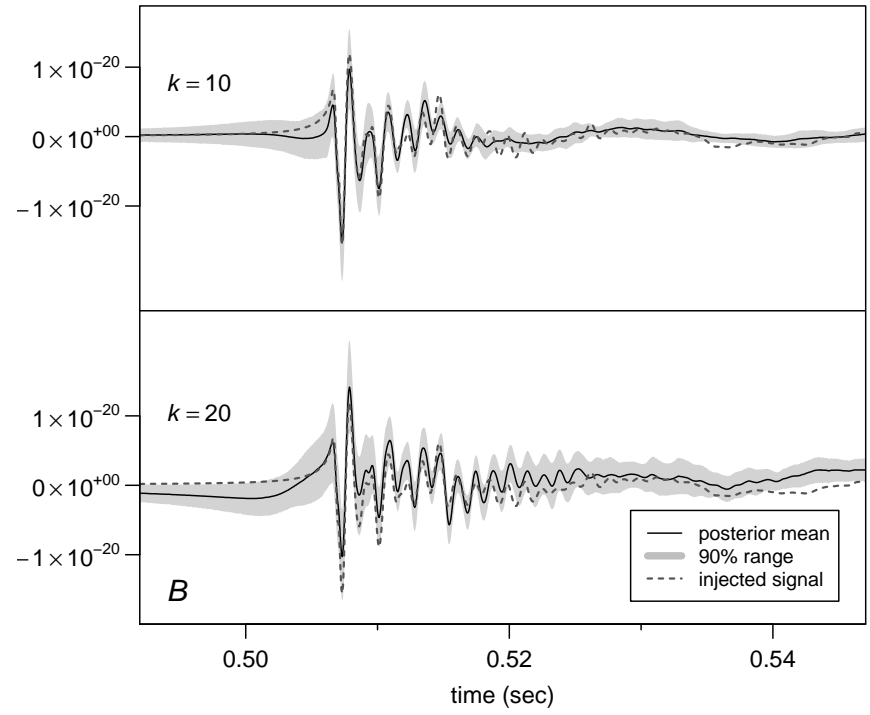

FIG. 6: A repeated analysis and reconstruction of the same example signal $B$ with SNR $\rho=10$ using different numbers of PCs $(k=10$ and $k=20)$. Note that a larger number of basis vectors in the model does not necessarily improve signal recovery.

three example waveforms from the previous section. The coefficients' posterior distribution can be related back to the sets of coefficients for reconstructing every signal in the original catalog, in order to identify similarities. A simple ad hoc measure of similarity is a $\chi^{2}$ match that relates a set of best-matching coefficients $\hat{\beta}_{i}(i=1, \ldots, k)$ associated with each catalog entry to the posterior distribution of the coefficients $\mathrm{P}\left(\beta_{i} \mid y\right)$ of the inferred signal. 


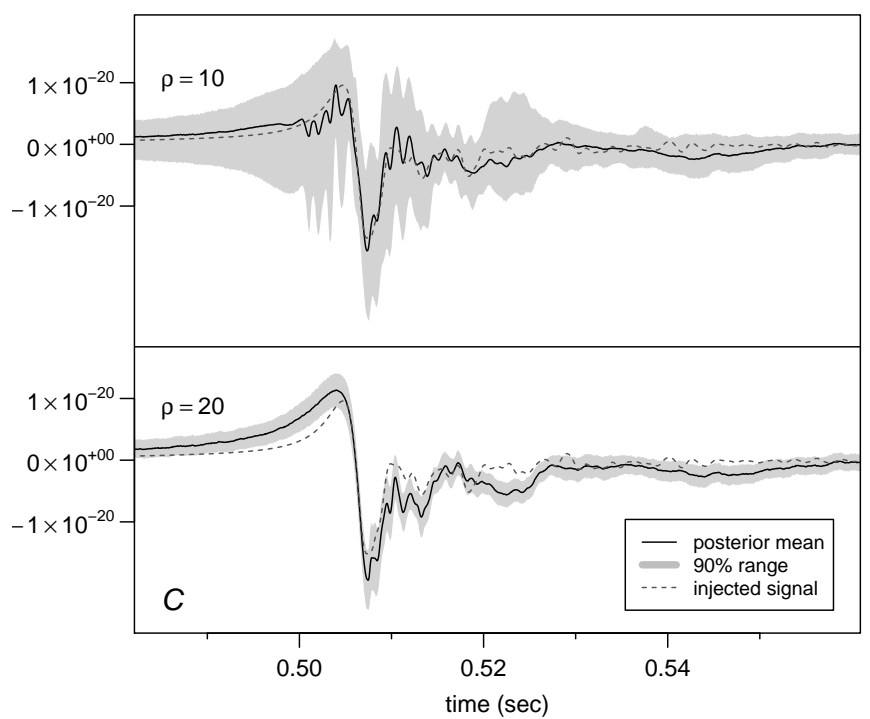

FIG. 7: Reconstruction of the same example signal $C$ at different overall amplitudes (and, with that, SNRs: $\rho=10$ and $\rho=20)$, both times using $k=10$ PCs.

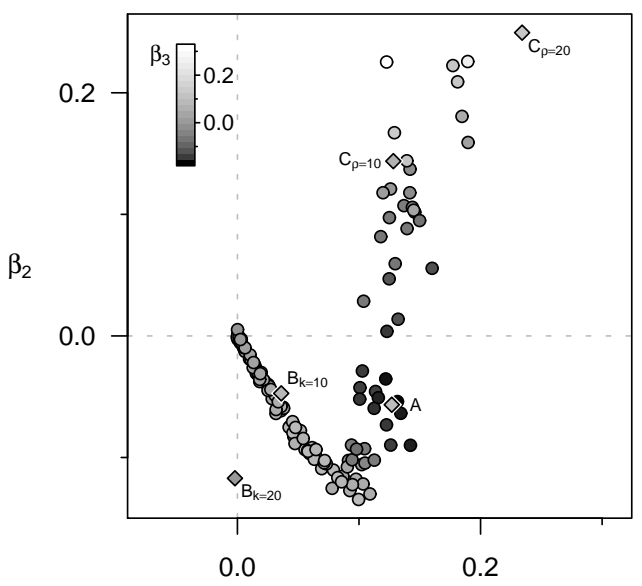

$\beta_{1}$

FIG. 8: The resulting first three PC coefficients $\beta_{1}, \beta_{2}$, and $\beta_{3}$ when matching each of the 128 waveforms in the catalog against the set of PCs (no noise involved). The waveforms in the catalog all correspond to a signal from a fixed distance $(10 \mathrm{kpc})$. Note that the coefficients only occupy a very restricted region in parameter space. The diamonds show the posterior mean values of the $\mathrm{PC}$ coefficients for the three examples (labeled A, B, and C) discussed in the text. For comparison they are scaled down (by their known distance) to the same magnitude.

Such a match may be defined as

$$
\chi^{2}=\min _{c \in \mathbb{R}} \sum_{i=1}^{k} \frac{\left(c \hat{\beta}_{i}-\mathrm{E}\left[\beta_{i} \mid \boldsymbol{y}\right]\right)^{2}}{\operatorname{Var}\left(\beta_{i} \mid \boldsymbol{y}\right)},
$$

considering only posterior means and variances of individual coefficients $\beta_{i}$, and allowing for a scaling factor $c$

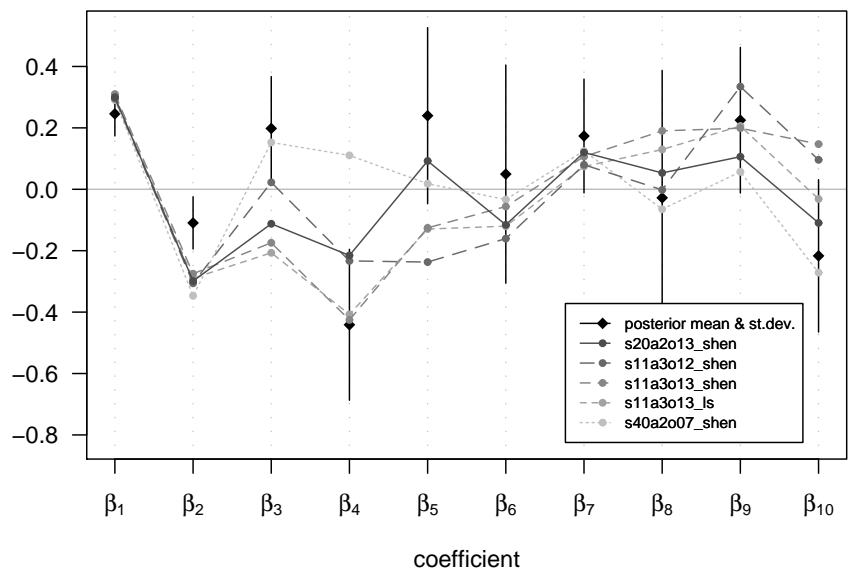

FIG. 9: Comparison of the distributions of all PC coefficients $\beta_{i}$ to the best-matched sets of coefficients found in the original catalog. Posterior means and standard deviations ('error bars') are shown in black, the (scaled) sets of the top five bestmatching coefficients are shown in different shades of gray.

in the overall amplitude of the matched signal. Computing the match for each signal from the catalog allows one to rank all signals and then determine those that fit the best. A mapping scheme set up this way combines elements of nearest-neighbor and naive-Bayes classification techniques [78].

For the initial example signal $A$ shown above (see Figs. (4 and 5), the posterior means and standard deviations ('error bars') are illustrated in a parallel coordinates plot 79] in Fig. 9, together with the five best-matching catalog entries. More details on the injected and the ten best-matching signals are given in Table I. Table Ilists the closest correspondents for the other example signals (examples $B$ and $C$ ) in comparison with the injection values. In the case of signal $A$, the best matching waveform from the catalog and the injected signal have very similar physical parameters. For signals $B$ and $C$ this simple method still provides a good estimation of the physical parameters, although admittedly not as accurate as case $A$; note that the reconstruction of the waveform is quite accurate for all three signals. We think that through the use of techniques such as Procrustes rotation [80] we will be able to provide a more direct link to the physical parameters. The posterior mean values for the first three PC coefficients $\left(\beta_{1}\right.$ to $\left.\beta_{3}\right)$ for all five examples are also illustrated in Fig. 8, Reconstruction of the measured signals through the PC basis vectors not only allows one to capture the the waveforms' appearances, but also yields information on the underlying physical parameters.

The information available from matching a signal candidate against a set of basis vectors may not only facilitate a classification within a set of possible astrophysical sources (as illustrated in Fig. 8), but may also help in distinguishing it from signals of different origin, like instrumental glitches, etc. Different types of potential 
TABLE I: The injected example signal $A$ and the corresponding top 10 best matching catalog entries. The column labeled with 'model' identifies the signal from the original catalog 69. The following columns state achieved $\chi^{2}$ match (according to Eq. (21)), the corresponding EoS, mass of the progenitor model $\left(M_{\mathrm{prog}}\right)$, precollapse angular velocity at the center $\left(\Omega_{\mathrm{c}, \mathrm{i}}\right)$, precollapse differential rotation length scale $(A)$, rotation rates initially $\left(T_{\mathrm{i}} /|W|_{\mathrm{i}}\right)$, at the time of bounce $\left(T_{\mathrm{b}} /|W|_{\mathrm{b}}\right)$, and late after bounce $\left(T_{\mathrm{pb}} /|W|_{\mathrm{pb}}\right)$, and the maximum density in the core at the time of bounce $\left(\rho_{\max , \mathrm{b}}\right)$ [21]. The corresponding best-matching effective distance $d_{\mathrm{e}}$ results from inverting the amplitude $c$ (see Eq. (21)) that yields the optimal match.

\begin{tabular}{|c|c|c|c|c|c|c|c|c|c|c|c|}
\hline rank & model & $\chi^{2}$ & EoS & $\begin{array}{c}M_{\text {prog }} \\
{\left[\mathrm{M}_{\odot}\right]} \\
\end{array}$ & $\begin{array}{c}\Omega_{\mathrm{c}, \mathrm{i}} \\
{\left[\mathrm{rad} \mathrm{s}^{-1}\right]}\end{array}$ & $\begin{array}{c}A \\
{[\mathrm{~km}]} \\
\end{array}$ & $\frac{T_{\mathrm{i}}}{|W|_{\mathrm{i}}}$ & $\begin{array}{c}\left|\frac{T_{\mathrm{b}}}{|W|_{\mathrm{b}}}\right| \\
{[\%]} \\
\end{array}$ & $\frac{T_{\mathrm{pb}}}{|W|_{\mathrm{pb}}}$ & $\begin{array}{c}\rho_{\max , \mathrm{b}} \\
{\left[10^{14} \mathrm{~g} \mathrm{~cm}^{-3}\right]}\end{array}$ & $\begin{array}{c}d_{\mathrm{e}} \\
{[\mathrm{kpc}]} \\
\end{array}$ \\
\hline & example signal $A$ & jected) & Shen & 20.0 & 5.48 & 1000 & 1.27 & 711.9 & 10.5 & 2.62 & 5.17 \\
\hline 1 & s20a2o13_shen & 10.8 & Shen & 20.0 & 6.45 & 1000 & 1.80 & | 14.8 & | 12.8 & 2.42 & 3.50 \\
\hline 2 & s11a3o12_shen & 12.8 & Shen & 11.2 & 10.65 & 500 & 1.28 & | 14.9 & | 12.3 & 2.46 & 3.46 \\
\hline 3 & s11a3o13_shen & 13.7 & Shen & 11.2 & 11.30 & 500 & 1.44 & | 16.1 & | 13.2 & 2.36 & 3.37 \\
\hline 4 & s11a3o13_1s & 13.8 & $\mathrm{LS}$ & 11.2 & 11.30 & 500 & 1.44 & $1 \mid 15.8$ & | 13.2 & 2.64 & 3.10 \\
\hline 5 & s40a2o07_shen & 14.6 & Shen & 40.0 & 3.40 & 1000 & 0.72 & $\mid 11.8$ & 9.9 & 2.58 & 3.75 \\
\hline 6 & s11a3o12_ls & 15.4 & $\mathrm{LS}$ & 11.2 & 10.65 & 500 & 1.28 & $\mid 14.7$ & | 12.3 & 2.75 & 3.12 \\
\hline 7 & s15a2o09_shen & 15.4 & Shen & 15.0 & 4.56 & 1000 & 1.09 & 11.8 & | 10.3 & 2.58 & 3.42 \\
\hline 8 & s20a3o09_shen & 15.7 & Shen & 20.0 & 8.99 & 500 & 0.90 & 15.7 & | 12.5 & 2.38 & 3.58 \\
\hline 9 & s20a3o07_shen & 17.4 & Shen & 20.0 & 5.95 & 500 & 0.50 & | 10.1 & 8.0 & 2.70 & 3.24 \\
\hline 10 & s20a2o13_1s & 20.6 & LS & 20.0 & 6.45 & 1000 & 1.80 & $\mid 14.4$ & 12.9 & 2.75 & 2.94 \\
\hline$\vdots$ & $\vdots$ & $\vdots$ & $\vdots$ & $\vdots$ & $\vdots$ & $\vdots$ & & : & & $\vdots$ & $\vdots$ \\
\hline
\end{tabular}

TABLE II: The injected signal example signals $B$ and $C$, and the corresponding best matching catalog entries when varying $k$ or $\rho$, respectively; see also Section VIB and Table $\square$

\begin{tabular}{|c|c|c|c|c|c|c|c|c|c|c|c|}
\hline PCs / SNR & model & $\chi^{2}$ & EoS & $\begin{array}{c}M_{\text {prog }} \\
{\left[\mathrm{M}_{\odot}\right]} \\
\end{array}$ & $\begin{array}{c}\Omega_{\mathrm{c}, \mathrm{i}} \\
{\left[\mathrm{rad} \mathrm{s}^{-1}\right]}\end{array}$ & $\begin{array}{c}A \\
{[\mathrm{~km}]}\end{array}$ & $\frac{T_{\mathrm{i}}}{|W|_{\mathrm{i}}} \mid$ & $\begin{array}{c}\frac{T_{\mathrm{b}}}{|W|_{\mathrm{b}}} \mid \\
{[\%]} \\
\end{array}$ & $\frac{T_{\mathrm{pb}}}{|W|_{\mathrm{pb}}}$ & $\begin{array}{c}\rho_{\max , \mathrm{b}} \\
{\left[10^{14} \mathrm{~g} \mathrm{~cm}^{-3}\right]}\end{array}$ & $\begin{array}{c}d_{\mathrm{e}} \\
{[\mathrm{kpc}]}\end{array}$ \\
\hline \multicolumn{3}{|c|}{ example signal B (injected) } & $\mathrm{LS}$ & 15.0 & 2.82 & 1000 & 0.41 & $|5.5|$ & 5.2 & 3.61 & 3.24 \\
\hline$k=10$ & s11a3o07_shen & 1.9 & Shen & 20.0 & 5.95 & 500 & $0.40 \mid$ & $|5.9|$ & 5.1 & 2.89 & 3.41 \\
\hline$k=20$ & s15a1013_1s & 47.4 & $\mathrm{LS}$ & 15.0 & 2.71 & 50000 & 3.26 & $6.1 \mid$ & 6.6 & 3.56 & 1.28 \\
\hline \multicolumn{3}{|c|}{ example signal $C$ (injected) } & LS & 40.0 & 9.60 & 500 & 1.46 & $|22.1|$ & 19.0 & 0.73 & $8.32 \mid 4.16$ \\
\hline$\rho=10$ & s40a3o13_1s & 5.8 & LS & 40.0 & 11.30 & 500 & $2.07 \mid$ & $|23.4|$ & 20.7 & 0.28 & 5.38 \\
\hline$\rho=20$ & s40a2o15_shen & 19.6 & Shen & 40.0 & 7.60 & 1000 & 3.62 & $|21.1|$ & 20.3 & 0.27 & 2.32 \\
\hline
\end{tabular}

signals may also be partly reconstructible in terms of a linear combination of PCs, but a poor match, an unusual combination of coefficients, or a better match from an alternative set of basis vectors may then provide evidence in favor or against certain signal origins.

\section{CONCLUSIONS AND OUTLOOK}

In this paper we have described initial work on implementing a scheme by which the physical parameters associated with a rotating collapse and bounce in a stellar core collapse event can be estimated through the observation of gravitational waves by detectors such as LIGO, VIRGO, GEO or TAMA. We have presented a technique which allows one to reconstruct the detected signal through the use of numerically calculated gravitational radiation waveforms, principal component analy- sis, and Markov chain Monte Carlo (MCMC) methods; we have then compared the reconstructed signals to the table of numerically calculated waveforms, and inferred the physical parameters.

The results displayed in this paper were achieved through the application of a number of different and difficult analysis techniques. We are encouraged that in this initial study we have displayed that these methods can be combined in a way such that physical information about the supernova event can be extracted from the detected gravitational wave data. We have found that our method is quite successful in the reconstruction of the detected waveform, and this is displayed in the three examples. In the present analysis we have used the output from a single detector, and we expect the accuracy of the signal reconstruction will only get better as we expand the application of this work to the coherent analysis of multiple interferometers. We were able to make a fairly good asso- 
ciation with the physical parameters; however, we think that we will be able to greatly improve on our ability to make parameter estimation estimates. In the present study we worked with a relatively small signal catalog, where each physical parameter took on only a handful of different values; for example, there were only two EoS to choose from. Our parameter estimation demonstration was pretty good within these constraints, but we expect the accuracy to increase with expanded signal catalogs. In addition, Procrustes rotation [80] is an example of one technique that we intend to apply in order to make better statistical estimates of the physical parameters based on the eigenstates provided by the PCA.

Our eventual goal is to apply the method to the output from a network of detectors, which will provide an even better ability to discriminate signal and noise, reconstruct the signal, plus allow us to estimate the source position on the sky. This would necessitate the use of catalogs for stellar core collapse waveforms which are both quantitatively more extensive and qualitatively improved, so that the parameter space of likely events can be completely covered and very finely resolved. For instance, a larger selection of available equation of state tables will extend the phase space in that respective direction, while a more accurate inclusion of neutrino effects will both improve the quality of the burst signal from core bounce and as well yield a much more appropriate characterization of the low-frequency signal from post-bounce convective bulk motion in the post-shock region and in the proto-neutron star. In addition, other prospective emission mechanisms for gravitational waves in a core collapse event, like proto-neutron star pulsations or nonaxisymmetric rotational instabilities, could be also considered (for a comprehensive review on various such mechanisms, see [18]).

The method presented in this paper will provide a better way to produce statistical and probabilistic statements about the physical parameters associated with a stellar core collapse event, and the ability to make statements about actual signals observed by interferometric detectors. The data from multiple interferometers will be coherently analyzed; this will allow the estimation of parameters associated with the location of the source on the sky, similar to what can be achieved with coherent MCMC analysis of binary inspiral gravitational wave data [29, 30]. As noted previously in our examples, the timing accuracy is better than $1 \mathrm{~ms}$ for a single interferometer observation; a multiple interferometer coherent MCMC should provide very good sky localization estimation, and the use of principal components within the framework of Searle et al. [39] also shows great promise. In addition, a coherent analysis will also allow us to better distinguish mismatch ( $\boldsymbol{m}$, common to all detectors) from noise ( $\boldsymbol{\epsilon}$, different between different detectors) and hence should improve the waveform reconstruction. We also plan to incorporate a flexible model for the detector noise spectrum, as described in [73]. A proper accounting for the noise spectral densities of the interferometers could also be applied in producing the PCA eigenvectors and eigenvalues, i.e. these would not simply be the result of a least-squares fit, but rather a noise-weighted least-squares fit. The resulting basis vectors should then better reflect what is actually "visible" to an interferometric detector within its limited sensitivity band.

An important part of our long-term research program will be to ensure that the catalog of waveforms truly spans and encompasses those from physically possible rotating stellar core collapses; it will be critical for us to consult closely with experts in the field [18, 19, 20, 21] to assure that the physical parameters for the waveforms we will use cover the range of natural possibilities. Typically when initially constructing the initial models one already has some idea about how many intermediate steps per free parameters are required. When the waveforms are generated as the output from the calculations (along with the other output data), then the variations of the waveforms and the other data give some indication if the original choice of model construction and parameter division are sensible. While the signal catalog needs to stretch over the extreme limits of the values for the physical parameters, a strength of our method is that the table need not be densely populated (as is the case with the template bank for coalescing binary signals); this the advantage provided by the use of the PCA. For example, when the PCA eigenvalues are derived, their variation provides guidance about the quality of our catalog. We can test whether the waveform table contains enough entries by successfully reconstructing additional (off-table) signals placed within the parameter space; this was essentially the method used for verifying the results presented in this article.

The technique described in this paper could prove to be advantageous for signal reconstruction and parameter estimation when numerical techniques are required in order to produce the waveforms. For example, we can imagine our method as being useful for estimating parameters for complicated binary inspiral signals; numerical relativity calculations are producing a wide array of complicated inspiral signals [81]. We have already shown in Section VII that the fitted PC coefficients can be related to the original physical parameters of the signals in the catalog. Future research will explore how the joint posterior distribution of the PC coefficients can be employed for statistical inference on the physical parameters by linking the different parameter spaces via Procrustes rotation [80].

This method should also prove to be useful in distinguishing a real stellar core collapse event from a common noise glitch in the data. As displayed in Fig. 8, there is a characteristic pattern to be found with PCA values associated with a real signal. A glitch produced by noise, when reconstructed via PCA, will likely fall outside the pattern formed by stellar core collapse signals. Alternatively, one could set up an alternative glitch model, which, instead of using numerical simulations for the (PCA) basis vector generation, is trained on sets of 
measured waveforms that are known to be instrumental artifacts. We intend to test this potential veto technique.

In this paper we have reduced the table of 128 waveforms to 10 or 20 of the most important PC eigenvectors. Instead of arbitrarily choosing the number of eigenvectors to use, we plan to have our MCMC optimize the signal reconstruction through the use of a reversible jump MCMC, similar to the method described in [82], thereby treating the number or subset of PCs as another unknown. In this Bayesian approach the optimal number of eigenvectors is determined by weighing the benefit of a good signal fit with a large number of eigenvectors against the Ockham's Razor penalization when the model gets overly complex.

\section{Acknowledgments}

It is a pleasure to thank Christian D. Ott and Patrick J. Sutton for a careful reading of the script. H.D. acknowledges a Marie Curie Intra-European Fellowship within the 6th European Community Framework Programme (IEF 040464). This work was supported by the MaxPlanck-Society, The Royal Society of New Zealand Marsden Fund grant UOA-204, National Science Foundation grants PHY-0553422 and PHY-0854790, the Fulbright Scholar Program, the DAAD and IKY (IKYDA GermanGreek research travel grant), by the European Network of Theoretical Astroparticle Physics ENTApP ILIAS/N6 under contract number RII3-CT-2004-506222, and by the Science and Technology Facilities Council of the United Kingdom and the Scottish Universities Physics Alliance.

\section{APPENDIX A: DEFINITION OF DISCRETE FOURIER TRANSFORM}

The discrete Fourier transform is defined for a realvalued function $h$ of time $t$, sampled at $N$ discrete time points at a sampling rate of $\frac{1}{\Delta_{t}}$.

The transform maps from

$$
\left\{h(t) \in \mathbb{R}: t=0, \Delta_{t}, 2 \Delta_{t}, \ldots,(N-1) \Delta_{t}\right\}
$$

to a function of frequency $f$

$$
\left\{\tilde{h}(f) \in \mathbb{C}: f=0, \Delta_{f}, 2 \Delta_{f}, \ldots,(N-1) \Delta_{f}\right\},
$$

where $\Delta_{f}=\frac{1}{N \Delta_{t}}$ and

$$
\tilde{h}(f)=\sum_{j=0}^{N-1} h\left(j \Delta_{t}\right) \exp \left(-2 \pi \mathrm{i} j \Delta_{t} f\right) .
$$

Since $h$ is real-valued, the elements of $\tilde{h}$ are symmetric in the sense that $\tilde{h}\left(i \Delta_{f}\right)$ and $\tilde{h}\left((N-i) \Delta_{f}\right)$ are complex conjugates; this allows to restrict attention to the nonredundant elements indexed by $i=0, \ldots, N / 2$.

APPENDIX B: FOURIER DOMAIN MODEL

A Gaussian distribution of a (time-domain) random vector also implies gaussianity for the Fourier transformed vector. In particular, if $n(t)$ is (zero mean, stationary) Gaussian noise with one-sided power spectral density $S_{1}(f)$, then in the limit of large $N$ and small $\Delta_{t}$ the real and imaginary components of the discrete Fourier transformed time series are independently zeromean Gaussian distributed:

$$
\mathrm{P}\left(\operatorname{Re}\left(\tilde{n}\left(f_{j}\right)\right)\right)=\mathrm{N}\left(0, \sigma_{f_{j}}^{2}\right), \quad \mathrm{P}\left(\operatorname{Im}\left(\tilde{n}\left(f_{j}\right)\right)\right)=\mathrm{N}\left(0, \sigma_{f_{j}}^{2}\right),
$$

where the variance parameter is $\sigma_{f_{j}}^{2}=\frac{N}{4 \Delta_{t}} S_{1}\left(f_{j}\right)$ [72, 73, 83.

\section{APPENDIX C: JOINT AND CONDITIONAL POSTERIORS}

For the random effects model from Sec. VC the joint distribution of data and parameters may be factored out into:

$$
\begin{aligned}
& \mathrm{p}\left(\boldsymbol{y}, \boldsymbol{\beta}, T, \sigma_{m}^{2}, \tilde{\boldsymbol{m}}\right) \\
= & \mathrm{p}\left(\boldsymbol{y} \mid \boldsymbol{\beta}, T, \sigma_{m}^{2}, \tilde{\boldsymbol{m}}\right) \times \mathrm{p}\left(\tilde{\boldsymbol{m}} \mid \boldsymbol{\beta}, T, \sigma_{m}^{2}\right) \\
& \times \mathrm{p}\left(\sigma_{m}^{2} \mid \boldsymbol{\beta}, T\right) \times \mathrm{p}(\boldsymbol{\beta}, T) \\
\propto & \exp \left(-2 \sum_{j=1}^{N / 2} \frac{\frac{\Delta_{t}}{N}\left|\tilde{y}_{j}-\left(\tilde{\boldsymbol{X}}_{(T)} \boldsymbol{\beta}\right)_{j}-\tilde{m}_{j}\right|^{2}}{S_{1}\left(f_{j}\right)}\right) \\
& \times\left(\sigma_{m}^{2}\right)^{-N / 2} \exp \left(-\sum_{i=1}^{N} \frac{m_{i}^{2}}{2 \sigma_{m}^{2}}\right) \\
& \times\left(\frac{1}{N}\|\boldsymbol{X} \boldsymbol{\beta}\|^{2}\right)^{\frac{\nu}{2}}\left(\sigma_{m}^{2}\right)^{-1-\frac{\nu}{2}} \exp \left(\frac{-\nu \gamma \frac{1}{N}\|\boldsymbol{X} \boldsymbol{\beta}\|^{2}}{2 \sigma_{m}^{2}}\right),
\end{aligned}
$$

where the proportionality here refers to keeping the data and known parameters constant. In the above equation, the first term (C2) is the 'usual' time-domain likelihood from Appendix $\mathrm{B}$, the term (C3) is the likelihood of the mismatch (random effect), and the term (C4) is a conditional prior of the mismatch variance parameter. By fixing the values for different subsets of parameters, one can determine conditional posterior distributions that are useful for the Gibbs sampling implementation (see Section VD). 
[1] A. Abramovici et al., Science 256, 325 (1992).

[2] B. C. Barish, in Gravitational wave detection: Proceedings of the TAMA international workshop on gravitational wave detection, edited by K. Tsubono et al. (Universal Academy Press, Tokyo, 1997), pp. 155-161.

[3] B. Abbott et al., Nucl. Instrum. Meth. A 517, 154 (2004).

[4] B. Caron et al., Nucl. Phys. B (Proc. Suppl.) 48, 107 (1996).

[5] A. Brillet, in Gravitational wave detection: Proceedings of the TAMA international workshop on gravitational wave detection, edited by K. Tsubono et al. (Universal Academy Press, Tokyo, 1997), pp. 163-173.

[6] J. Hough et al., in Gravitational wave detection: Proceedings of the TAMA international workshop on gravitational wave detection, edited by K. Tsubono et al. (Universal Academy Press, Tokyo, 1997), pp. 175-182.

[7] K. Tsubono et al., in Gravitational wave detection: Proceedings of the TAMA international workshop on gravitational wave detection, edited by K. Tsubono et al. (Universal Academy Press, Tokyo, 1997), pp. 183-191.

[8] J. R. Smith et al., Class. Quantum Grav. 26, 114013 (2009).

[9] URL: http://www.ligo.caltech.edu/advLIGO/

[10] F. Acernese et al., J. Opt. A 10, 064009 (2008).

[11] URL: http://wwwcascina.virgo.infn.it/advirgo/.

[12] F. Beauville et al., Class. Quantum Grav. 25, 045002 (2008).

[13] B. Abbott et al., Class. Quantum Grav. 24 (2007).

[14] B. Abbott et al., Class. Quantum Grav. 25, 245008 (2008).

[15] B. Abbott et al., Phys. Rev. D 72, 122004 (2005).

[16] F. Acernese et al., Class. Quantum Grav. 26, 085009 (2009).

[17] M. Ando et al., Phys. Rev. D 71, 082002 (2005).

[18] C. D. Ott, Class. Quantum Grav. 26, 063001 (2009).

[19] C. D. Ott, H. Dimmelmeier, A. Marek, H.-T. Janka, I. Hawke, B. Zink, and E. Schnetter, Phys. Rev. Lett. 98, 261101 (2007).

[20] H. Dimmelmeier, C. D. Ott, H. T. Janka, A. Marek, and E. M. Müller, Phys. Rev. Lett. 98, 251101 (2007).

[21] H. Dimmelmeier, C. D. Ott, A. Marek, and H.-T. Janka, Phys. Rev. D 78, 064056 (2008).

[22] L. S. Finn, Arxiv preprint gr-qc/9709077 (1997).

[23] W. R. Gilks, S. Richardson, and D. J. Spiegelhalter, Markov chain Monte Carlo in practice (Chapman \& Hall / CRC, Boca Raton, 1996).

[24] T. J. Loredo, in Statistical challenges in modern astronomy, edited by E. D. Feigelson and G. J. Babu (SpringerVerlag, New York, 1992), chap. 12, pp. 275-297.

[25] P. C. Gregory, in Bayesian inference and Maximum Entropy methods in science and engineering, edited by A. Mohammad-Djafari (American Institute of Physics, 2001), vol. 568 of AIP Conference Proceedings, pp. 557568.

[26] N. Christensen and R. Meyer, Phys. Rev. D 64, 022001 (2001).

[27] N. Christensen, R. Meyer, and A. Libson, Class. Quantum Grav. 21, 317 (2004).

[28] C. Röver, R. Meyer, and N. Christensen, Class. Quantum Grav. 23, 4895 (2006).

[29] C. Röver, R. Meyer, and N. Christensen, Phys. Rev. D
75, 062004 (2007).

[30] C. Röver, R. Meyer, G. M. Guidi, A. Viceré, and N. Christensen, Class. Quantum Grav. 24, S607 (2007).

[31] M. V. van der Sluys, C. Röver, A. Stroeer, N. Christensen, V. Kalogera, R. Meyer, and A. Vecchio, Astrophys. J. Lett. 688, L61 (2008).

[32] N. Christensen, R. J. Dupuis, G. Woan, and R. Meyer, Phys. Rev. D 70, 022001 (2004).

[33] R. Umstätter, R. Meyer, R. Dupuis, J. Veitch, G. Woan, and N. Christensen, Class. Quantum Grav. 21, S1655 (2004).

[34] J. Veitch, R. Umstätter, R. Meyer, N. Christensen, and G. Woan, Class. Quantum Grav. 22, S995 (2005).

[35] I. S. Heng, Class. Quantum Grav. 26, 105005 (2009).

[36] Y. Gürsel and M. Tinto, Phys. Rev. D 40, 3884 (1989).

[37] M. Rakhmanov, Class. Quantum Grav. 23, S673 (2006).

[38] J. S. Key and N. J. Cornish, Phys. Rev. D 79, 043014 (2009).

[39] A. C. Searle, P. J. Sutton, and M. Tinto, Class. Quantum Grav. 26, 155017 (2009).

[40] T. Z. Summerscales, A. Burrows, L. S. Finn, and C. D. Ott, Astrophys. J. 678, 1142 (2008).

[41] C. D. Ott, A. Burrows, E. Livne, and R. Walder, Astrophys. J. 600, 834 (2004).

[42] W. G. Anderson, P. R. Brady, J. D. E. Creighton, and E. E. Flanagan, Phys. Rev. D 63, 042003 (2001).

[43] S. Klimenko and G. Mitselmakher, Class. Quantum Grav. 21, S1819 (2004).

[44] S. Chatterji, L. Blackburn, G. Martin, and E. Katsavounidis, Class. Quantum Grav. 21, S1809 (2004).

[45] S. Chatterji, Ph.D. thesis, Massachussetts Institute of Technology (2005).

[46] A.-C. Clapson, M. Barsuglia, M.-A. Bizouard, V. Brisson, F. Cavalier, M. Davier, P. Hello, N. Leroy, and M. Varvella, Class. Quantum Grav. 25, 035002 (2008).

[47] J. Sylvestre, Phys. Rev. D 68, 102005 (2003).

[48] N. Arnaud, M. Barsuglia, M.-A. Bizouard, V. Brisson, F. Cavalier, M. Davier, P. Hello, S. Kreckelbergh, and E. K. Porter, Phys. Rev. D 67, 102003 (2003).

[49] L. Wen and B. Schutz, Class. Quantum Grav. 22, S1321 (2005).

[50] S. Mohanty, M. Rakhmanov, S. Klimenko, and G. Mitselmakher, Class. Quantum Grav. 23, 4799 (2006).

[51] S. Klimenko, I. Yakushin, A. Mercer, and G. Mitselmakher, Class. Quantum Grav. 25, 114029 (2008).

[52] P. Sutton et al., Arxiv preprint 0908.3665 [gr-qc] (2009).

[53] F. Cavalier et al., Phys. Rev. D 74, 082004 (2006).

[54] J. Markowitz, M. Zanolin, L. Cadonati, and E. Katsavounidis, Phys. Rev. D 78, 122003 (2008).

[55] S. Fairhurst, Arxiv preprint 0908.2356 [gr-qc] (2009).

[56] L. Baiotti, I. Hawke, L. Rezzolla, and E. Schnetter, Phys. Rev. Lett. 94, 131101 (2005).

[57] E. E. Flanagan and S. A. Hughes, Phys. Rev. D 57, 4566 (1998).

[58] H. Dimmelmeier, J. A. Font, and E. Müller, Astron. Astrophys. 393, 523 (2002).

[59] H. Dimmelmeier, J. Novak, J. A. Font, J. M. Ibáñez, and E. Müller, Phys. Rev. D 71, 064023 (2005).

[60] J. A. Isenberg, Int. J. Mod. Phys. D 17, 265 (2008).

[61] A. Heger, N. Langer, and S. E. Woosley, Astrophys. J. 528, 368 (2000). 
[62] A. Heger, S. E. Woosley, and H. C. Spruit, Astrophys. J. 626, 350 (2005).

[63] H. Shen, H. Toki, K. Oyamatsu, and K. Sumiyoshi, Prog. Theor. Phys. 100, 1013 (1998).

[64] H. Shen, H. Toki, K. Oyamatsu, and K. Sumiyoshi, Nucl. Phys. A 637, 435 (1998).

[65] J. M. Lattimer, C. J. Pethick, D. G. Ravenhall, and D. Q. Lamb, Nucl. Phys. A 432, 646 (1985).

[66] J. M. Lattimer and F. D. Swesty, Nucl. Phys. A 535, 331 (1991).

[67] A. Marek, H.-T. Janka, R. Buras, M. Liebendörfer, and M. Rampp, Astron. Astrophys. 443, 201 (2005).

[68] M. Liebendörfer, Astrophys. J. 633, 1042 (2005).

[69] URL www mpa-garching.mpg.de/rel hydro/wave_catalog sBom. V.

[70] S. E. Woosley and A. Heger, Astrophys. J. 637, 914 (2006).

[71] G. Strang, Introduction to Linear Algebra (Wellesley Cambridge Press, Wellesley, MA, 1993).

[72] L. S. Finn, Phys. Rev. D 46, 5236 (1992).

[73] C. Röver, R. Meyer, and N. Christensen, Arxiv preprint 0804.3853 (2008).

[74] G. L. Bretthorst, in Maximum Entropy and Bayesian Methods, edited by W. v. d. Linden et al. (Kluwer Academic Publishers, Dordrecht, The Netherlands, 1999), pp. $21-46$.

[75] A. Gelman, J. B. Carlin, H. Stern, and D. B. Rubin, Bayesian data analysis (Chapman \& Hall / CRC, Boca Raton, 1997).

[76] T. Damour, B. R. Iyer, and B. S. Sathyaprakash, Phys. Rev. D 63, 044023 (2001).

[77] J. Creighton et al., LAL software documentation (2007), URL http://www. lsc-group.phys.uwm.edu/daswg/projects/lal.html

[78] T. Hastie, R. Tibshirani, and J. Friedman, The elements of statistical learning; data mining, inference, and prediction (Springer-Verlag, New York, 2001).

[79] A. Inselberg, Visual Comput. 1, 69 (1985).

Analysis (Academic Press, London, 1979).

[81] B. Aylott et al., Class. Quantum Grav. 26, 165008 (2009).

[82] R. Umstätter, N. Christensen, M. Hendry, R. Meyer, V. Simha, J. Veitch, S. Vigeland, and G. Woan, Phys. Rev. D 72, 022001 (2005).

[83] D. R. Brillinger, Time Series: Data Analysis and Theory (McGraw-Hill, New York, 1981). 